\title{
DATOS SOBRE LA VEGETACIÓN DE LOS LLANOS OCCIDENTALES DEL ORINOCO (VENEZUELA)
}

\author{
Antonio GALÁN DE MERA, Adolfo GONZÁLEZ, Raquel MORALES, \\ Beatriz OLTRA y José A. VICENTE ORELLANA
}

\begin{abstract}
RESUMEN. Datos sobre la vegetación de los Llanos Occidentales del Orinoco (Venezuela). Los Llanos Occidentales del Orinoco se caracterizan por ser una gran cuenca sedimentaria donde se alternan sustratos arcillosos y arenosos. El bioclima infratropical subhúmedo y la cantidad de ambientes palustres originan una gran diversidad de comunidades vegetales, en especial de helófitos, pleustohelófitos e hidrófitos. Cuando la inundación es más escasa, aparecen los bosques, arbustedas y los pastos secos de la sabana, que forman isleos de comunidades vegetales en medio de la llanura inundable. Hemos podido reconocer 17 asociaciones repartidas en 10 clases fitosociológicas: CabomboNymphaeetea (comunidades de hidrófitos), Ceibetea occidentalis (bosques y arbustedas), Cladietea jamaicensis (comunidades de helófitos), Coccolobietea obtusifoliae (arbustedas riparias), Lemnetea minoris (comunidades de pleustófitos), Leptocoryphio-Trachypogonetea (pastizales secos de la sabana), Pistio stratiotidis-Eichhornietea crassipedis (comunidades de pleustohelófitos), Polygono arenastri-Poetea annuae (comunidades nitrófilas resistentes al pisoteo), Sido-Stachytarphetaetea (comunidades nitrófilas) y Xyridetea savanensis (pastizales temporalmente inundados con plantas anuales). Además, describimos como novedades sintaxonómicas 4 asociaciones (Bromelio chrysanthaePlatymiscietum pinnati, Geophiletum repentis, Helictero guazumifoliae-Bauhinietum benthamianae y Randio venezuelensis-Annonetum jahnii), 6 alianzas (Coccolobion obtusifoliae, Desmonco orthacanthi-Platymiscion pinnati, Heterantherion reniformis, Nectandro globosae-Viticion orinocensis, Oryzion perennis y Wissadulo periplocifoliae-Cassion torae), 3 órdenes (Bactrido guineensis-Cecropietalia peltatae, Coccolobietalia obtusifoliae y Eleocharitetalia minimae) y 1 clase (Coccolobietea obtusifoliae).
\end{abstract}

Palabras clave. Vegetación, sintaxonomía, Llanos, Venezuela.

\begin{abstract}
Data about the vegetation of the Occidental Llanos of the Orinoco (Venezuela). The western Llanos of the Orinoco river are a sedimentary basin of sandy and clayey mixed soils. The infratropical subhumid bioclimate and the large marshes cause a high diversity of plant communities consisting specially of helophytes, pleustohelophytes and hydrophytes. When there is a little flooding, forest, shrubs and dry pastures of the savannas appear, forming islands of plant communities in the swampy plain. Seventeen associations belonging to 10 classes have been recognized (CabomboNymphaeetea (hydrophytic communities), Ceibetea occidentalis (forests and shrubs), Cladietea jamaicensis (helophytic communities), Coccolobietea obtusifoliae (riparian shrubs), Lemnetea minoris (pleustophytic communities), Leptocoryphio-Trachypogonetea (dry pastures of the savanna), Pistio stratiotidis-Eichhornietea crassipedis (pleustohelophytic communities), Polygono arenastri-Poetea annuae (nitrophilous communities adapted to treading), Sido-Stachytarphetaetea (nitrophilous communities) and Xyridetea savanensis (temporal flooded pastures with annual plants). Moreover, 4 new associations (Bromelio chrysanthae-Platymiscietum pinnati, Geophiletum repentis, Helictero guazumifoliae-Bauhinietum benthamianae and Randio venezuelensis-Annonetum jahnii), 6 alliances (Coccolobion obtusifoliae, Desmonco orthacanthi-Platymiscion pinnati, Heterantherion reniformis, Nectandro globosae-Viticion orinocensis, Oryzion perennis y Wissadulo periplocifoliae-Cassion torae),
\end{abstract}


3 orders (Bactrido guineensis-Cecropietalia peltatae, Coccolobietalia obtusifoliae and Eleocharitetalia minimae) and 1 class (Coccolobietea obtusifoliae) are described.

Key words. Vegetation, syntaxonomy, Llanos, Venezuela.

\section{INTRODUCCIÓN Y OBJETIVOS}

Los Llanos del Orinoco son una inmensa llanura que está situada al E de la cordillera de los Andes y al S de la cordillera costera venezolana. Su escasa altitud -50 $130 \mathrm{~m}$ - hace que estén recorridos por multitud de ríos y afluentes tributarios del río Orinoco.

La vegetación que ocupa esta llanura es un mosaico entre sabanas, humedales y bosques (Hueck, 1966), muchos de ellos alterados por la presión humana para crear amplios espacios de pastos para la ganadería (Plonczak, 1989; Seibert, 1996).

Con excepción de algunos trabajos fitosociológicos (Castroviejo \& López, 1985; Colonnello et al., 1986; Susach Campalans, 1989), que dan una idea sobre la diversidad de las comunidades vegetales de los Llanos del Orinoco, hasta ahora sólo existen listados florísticos y descripciones fisionómicas de la vegetación (Ramia, 1959, 1967; Blydenstein, 1961, 1962, 1963; Aristeguieta, 1968a, 1968b; Castillo, 1977; Cuello et al., 1989). El trabajo de Castroviejo \& López (1985) es pionero al iniciar la descripción de las asociaciones de los Llanos Occidentales, aunque no profundiza en la relación biogeográfica existente con otras regiones de América Central y del Sur; Susach Campalans (1989) aborda el estudio fitosociológico de los pastizales de los Llanos Centrales, y Colonnello et al. (1986) describen numerosas asociaciones en los Llanos Orientales.

El objetivo de este trabajo es la descripción de las comunidades vegetales en los Llanos Occidentales del Orinoco tomando como base la comparación con otras unidades ya descritas en América Central y del Sur.

\section{METODOLOGÍA}

\section{Datos bioclimáticos sobre los Llanos del Orinoco}

La metodología bioclimática propuesta por Rivas-Martínez et al. (1999) muestra bastante bien el paralelismo existente entre la vegetación y los intervalos del índice de termicidad (It) y el índice ombrotérmico (Io). En la tabla 1 mostramos las variables, índices bioclimáticos y la descripción bioclimática de las principales estaciones meteorológicas de Venezuela, las cuales señalamos en el mapa de la figura 1. Los datos meteorológicos han sido obtenidos de Müller (1982) y Rivas-Martínez (2005).

En los Llanos del Orinoco tenemos las estaciones de Arauca (10) (Colombia) y San Fernando de Apure (11) (Venezuela) con bioclima infratropical subhúmedo, con un período máximo de lluvias entre abril y octubre (Walter y Medina, 1971); sin embargo, Ciudad Bolívar (6), situada al este, presenta un intervalo de lluvias seco. Esto quiere decir que existe un gradiente de humedad de oeste a este que se manifiesta en la vegetación (Aristeguieta, 1968a; Sarmiento \& Monasterio, 1969; Cuello et al., 1989) pudiéndose diferenciar: 1) Llanos Occidentales con bosques semidecíduos, pastos con bastante humedad a lo largo del año, y con una gran diversidad de comunidades acuáticas, y 2) Llanos Orientales, más secos, con bosques decíduos y sabanas arenosas, que se extienden hasta la zona del delta del Orinoco. 


\begin{tabular}{|c|c|c|c|c|c|c|c|}
\hline Estación Meteorológica & $\mathrm{T}$ & M & $\mathrm{m}$ & $\mathrm{P}$ & It & Io & Descripción bioclimática \\
\hline $\begin{array}{l}\text { 1- Maracaibo, } 48 \mathrm{~m} \\
\left(10^{\circ} 39^{\prime} \mathrm{N}, 71^{\circ} 36^{\prime} \mathrm{O}\right)\end{array}$ & 29 & 32,2 & 22,8 & 571 & 838 & 1,7 & Infratropical semiárido \\
\hline $\begin{array}{l}\text { 2- Puerto Cabello, } 10 \mathrm{~m} \\
\left(10^{\circ} 28^{\prime} \mathrm{N}, 68^{\circ} 04^{\prime} \mathrm{O}\right)\end{array}$ & 27 & 29,4 & 22,2 & 882 & 785 & 2,7 & Infratropical seco \\
\hline $\begin{array}{l}\text { 3- Maiquetía, } 70 \mathrm{~m} \\
\left(10^{\circ} 37^{\prime} \mathrm{N}, 66^{\circ} 59^{\prime} \mathrm{O}\right)\end{array}$ & 27 & 27,8 & 22,2 & 558 & 766 & 1,7 & Infratropical semiárido \\
\hline $\begin{array}{l}\text { 4- Barcelona, } 7 \mathrm{~m} \\
\left(10^{\circ} 07^{\prime} \mathrm{N}, 64^{\circ} 41^{\prime} \mathrm{O}\right)\end{array}$ & 26 & 25,2 & 25,2 & 629 & 768 & 2,0 & Infratropical semiárido \\
\hline $\begin{array}{l}\text { 5- Guiria, } 13 \mathrm{~m} \\
\left(10^{\circ} 35^{\prime} \mathrm{N}, 62^{\circ} 19^{\prime} \mathrm{O}\right)\end{array}$ & 27 & 28,9 & 22,4 & 898 & 781 & 2,8 & Infratropical seco \\
\hline $\begin{array}{l}\text { 6- Ciudad Bolívar, } 60 \mathrm{~m} \\
\left(08^{\circ} 07^{\prime} \mathrm{N}, 63^{\circ} 32^{\prime} \mathrm{O}\right)\end{array}$ & 28 & 32,2 & 22,2 & 973 & 826 & 2,9 & Infratropical seco \\
\hline $\begin{array}{l}\text { 7- Tumeremo, } 187 \mathrm{~m} \\
\left(07^{\circ} 18^{\prime} \mathrm{N}, 61^{\circ} 27^{\prime} \mathrm{O}\right)\end{array}$ & 26 & 30,0 & 20,0 & 1236 & 762 & 3,9 & Infratropical subhúmedo \\
\hline $\begin{array}{l}8 \text { - San Antonio, } 404 \mathrm{~m} \\
\left(07^{\circ} 50^{\prime} \mathrm{N}, 72^{\circ} 26^{\prime} \mathrm{O}\right)\end{array}$ & 27 & 28,9 & 20,0 & 757 & 658 & 2,0 & Infratropical seco \\
\hline $\begin{array}{l}\text { 9- Mérida, } 1495 \mathrm{~m} \\
\left(08^{\circ} 35^{\prime} \mathrm{N}, 71^{\circ} 10^{\prime} \mathrm{O}\right)\end{array}$ & 18,8 & 22,8 & 13,3 & 1170 & 549 & 7,8 & Termotropical húmedo \\
\hline $\begin{array}{l}\text { 10- Arauca (Colombia), } 122 \mathrm{~m} \\
\left(07^{\circ} 04^{\prime} \mathrm{N}, 70^{\circ} 14^{\prime} \mathrm{O}\right)\end{array}$ & 27 & 29,4 & 21,7 & 1758 & 779 & 5,6 & Infratropical subhúmedo \\
\hline $\begin{array}{l}11 \text { - San Fernando de Apure, } \\
73 \mathrm{~m}\left(07^{\circ} 53^{\prime} \mathrm{N}, 67^{\circ} 26^{\prime} \mathrm{O}\right)\end{array}$ & 27 & 25,5 & 25,5 & 1491 & 781 & 4,6 & Infratropical subhúmedo \\
\hline $\begin{array}{l}\text { 12- Puerto Ayacucho, } 73 \mathrm{~m} \\
\left(05^{\circ} 36^{\prime} \mathrm{N}, 67^{\circ} 30^{\prime} \mathrm{O}\right)\end{array}$ & 27 & 27,7 & 23,2 & 2183 & 777 & 6,8 & Infratropical húmedo \\
\hline $\begin{array}{l}\text { 13- Santa Elena de Uairén, } \\
907 \mathrm{~m}\left(04^{\circ} 36^{\prime} \mathrm{N}, 61^{\circ} 07^{\prime} \mathrm{O}\right)\end{array}$ & 22 & 27,8 & 15,0 & 1796 & 651 & 6,7 & Termotropical húmedo \\
\hline $\begin{array}{l}\text { 14- San Carlos de Río Negro, } \\
95 \mathrm{~m}\left(01^{\circ} 45^{\prime} \mathrm{N}, 67^{\circ} 03^{\prime} \mathrm{O}\right)\end{array}$ & 26 & 25,4 & 25,4 & 3521 & 769 & 11,2 & Infratropical húmedo \\
\hline
\end{tabular}

Tabla 1. Variables climáticas, índices bioclimáticos y descripción bioclimática de estaciones meteorológicas de Venezuela, de acuerdo con la metodología de Rivas-Martínez (1999)[T: temperatura media anual $\left({ }^{\circ} \mathrm{C}\right), \mathrm{M}$ : temperatura media de las máximas del mes más frío $\left({ }^{\circ} \mathrm{C}\right), \mathrm{m}$ : temperatura media de las mínimas del mes más frío $\left({ }^{\circ} \mathrm{C}\right), \mathrm{P}$ : precipitación media anual $(\mathrm{mm})$, It: índice de termicidad -It $=(\mathrm{T}+\mathrm{M}+\mathrm{m}) 10-$, Io: índice ombrotérmico $-\mathrm{Io}=\mathrm{P} / \mathrm{T}-;$ pisos bioclimáticos: $\mathrm{It}=711-890 \rightarrow$ infratropical, $491-710 \rightarrow$ termotropical, 321-490 $\rightarrow$ mesotropical, 161-320 $\rightarrow$ supratropical, $<160 \rightarrow$ orotropical; intervalos ombrotérmicos: Io $=<0.2 \rightarrow$ ultrahiperárido, $0.2-0.4 \rightarrow$ hiperárido, $0.4-1.0 \rightarrow$ árido, $1.0-$ $2.0 \rightarrow$ semiárido, 2.0-3.6!í seco, 3.6-6.0 $\rightarrow$ subhúmedo, 6.0-12.0 $\rightarrow$ húmedo, 12.0-24.0 $\rightarrow$ hiperhúmedo, $>24.0 \rightarrow$ ultrahiperhúmedo]. Climatic variables, bioclimatic indices and bioclimatic description of meteorological stations of Venezuela according to the Rivas-Martinez's methodology (1999)[T: mean annual temperature $\left({ }^{\circ} \mathrm{C}\right)$, $\mathrm{M}$ : mean higuest temperatures of the coldest month $\left({ }^{\circ} \mathrm{C}\right), \mathrm{m}$ : mean lowest temperatures of the coldest month $\left({ }^{\circ} \mathrm{C}\right)$, $P$ : mean annual precipitation $(\mathrm{mm}), \mathrm{It}$ : thermicity index $-\mathrm{It}=$ $(T+M+m) 10-$, Io $=$ ombrothermic index $-I o=P / T-;$ bioclimatic belts: It $=711-890 \rightarrow$ infratropical, 491-710 $\rightarrow$ thermotropical, 321-490 $\rightarrow$ mesotropical, 161-320 $\rightarrow$ supratropical, $<160 \rightarrow$ orotropical; ombrothermic intervals: $I o=<0.2 \rightarrow$ ultrahyperarid, $0.2-0.4 \rightarrow$ hyperarid, $0.4-1.0 !$ i arid, 1.0-2.0 $\rightarrow$ semiarid, 2.0-3.6 $\rightarrow$ dry, 3.6-6.0 $\rightarrow$ subhumid, 6.0-12.0!í humid, 12.0-24.0!í hyperhumid, $>24.0 \rightarrow$ ultrahyperhumid].

\section{Datos biogeográficos sobre los Llanos del Orinoco}

Los Llanos del Orinoco presentan una buena cantidad de plantas en común con la Amazonía y con otros bosques húmedos del norte de Sudamérica y de América Central [p.ej. Arrabidaea mollissima (H.B.K.) Bureau \& K. Schum., Cissampelos pareira L., Combretum laxum Jacq., Copaifera officinalis (Jacq.) L., Monstera adansonii Schott, Platymiscium pinnatum (Jacq.) Dugand, Smilax cumanensis Humb. \& Bonpl. ex Willd., Strychnos panamensis 


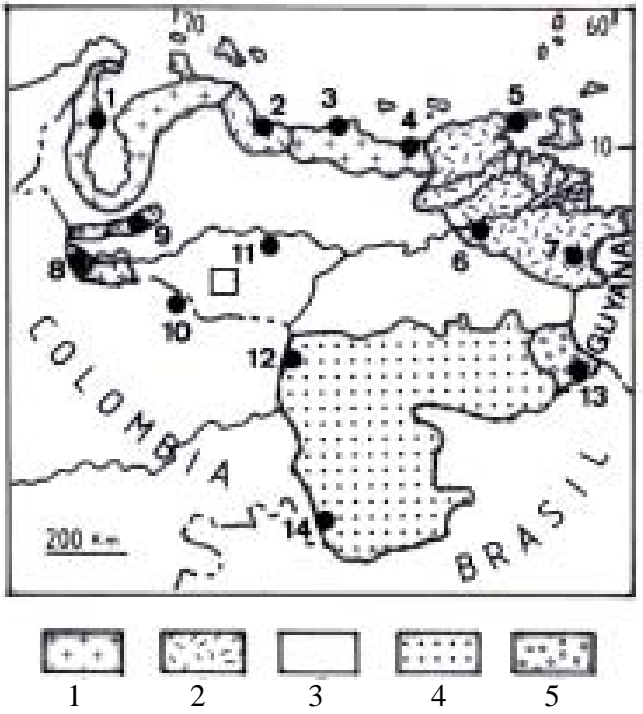

Figura 1. Mapa con una síntesis bioclimática de Venezuela (el cuadrado corresponde con el área de estudio y los puntos negros con las estaciones meteorológicas de la tab. 1). Map with a bioclimatic synthesis of Venezuela (the square is the study area and the black points are the meteorological stations of the tab. 1). 1Infratropical semiárido, 2- Infratropical seco, 3Infratropical subhúmedo, 4- Infratropical húmedo, 5- Termotropical húmedo.

Seem.]. Sin embargo, especies cuyas familias están muy representadas en la Amazonía no llegan a los Llanos.

Según la flora que estamos escribiendo sobre los Llanos Occidentales, distinguimos por el momento 10 endemismos [Borreria aristeguietana SÜÉлerm., Connarus venezuelanus var. orinocensis Forero, Duguetia riberensis Aristeg. ex Maas \& Boon, Caladium macrotites Schott, Gonolobus aristolochioides H.B.K., Hymenocallis venezuelensis Traub, Hyptis conferta var. angustata (Briq.) Pool \& Harley, Ipomoea pittieri O’Donell, Mitracarpus diffusus (Willd. ex Roem. \& Schult.) Cham. \& Schltdl. y Randia venezuelensis Steyerm.], que junto a la naturaleza sedimentaria de la llanura, su ombroclima subhúmedo frente al húmedo amazónico, y a sus confines geográficos, dibujan los límites de la provincia de los Llanos.

En el mapa de la figura 2 se señala la distribución de Annona jahnii Saff., Duguetia riberensis, Randia venezuelensis y Coccoloba obtusifolia Jacq. Aunque A. jahnii se encuentra también en la Guayana venezolana, es en los Llanos donde forma arbustedas con el endemismo $R$. venezuelensis; D. riberensis es un árbol propio de suelos inundables que no alcanza la región Amazónica e igualmente $C$. obtusifolia forma arbustedas que separan los territorios caribeño-mesoamericanos de la Amazonía. Por el contrario, Couratari gloriosa Sandwith, C. oblongifolia Ducke \& R. Knuth y Rourea cuspidata Benth. ex Baker son amazónicas y no alcanzan los Llanos (Prance, 1973, 1977), por lo que pensamos que el límite $\mathrm{S}$ de la provincia biogeográfica de los Llanos lo marcan las elevaciones de Meta y la Guainía, al E de Colombia. En Colombia, al SW de los Llanos, en la región de Araracuara, la vegetación característica de los Llanos Occidentales es sustituida por las arbustedas de Bonnetia martiana Maguire, Dimorphandra cuprea Sprague \& Sandwith e Ilex divaricata Mart. ex Reiss., y las sabanas de Axonopo schultesiSchoenocephalietum martiani Duivenvoorden \& Cleef 1994, relacionadas con la vegetación de la Gran Sabana y las "caatingas" amazónicas (Anderson et al., 1975; Duivenwoorden \& Cleef, 1994).

Tanto al O como al N, los Llanos dejan paso entre 200 y $500 \mathrm{~m}$ a la superprovincia del Páramo (Galán de Mera et al., 2002) y a la provincia Costera Venezolana (Huber, 1994) formando parte de la región CaribeñoMesoamericana (Rivas-Martínez et al., 1999; Morrone, 2001) lo cual puede justificarse por los bosques con una combinación de elementos comunes con los caribeños, como 


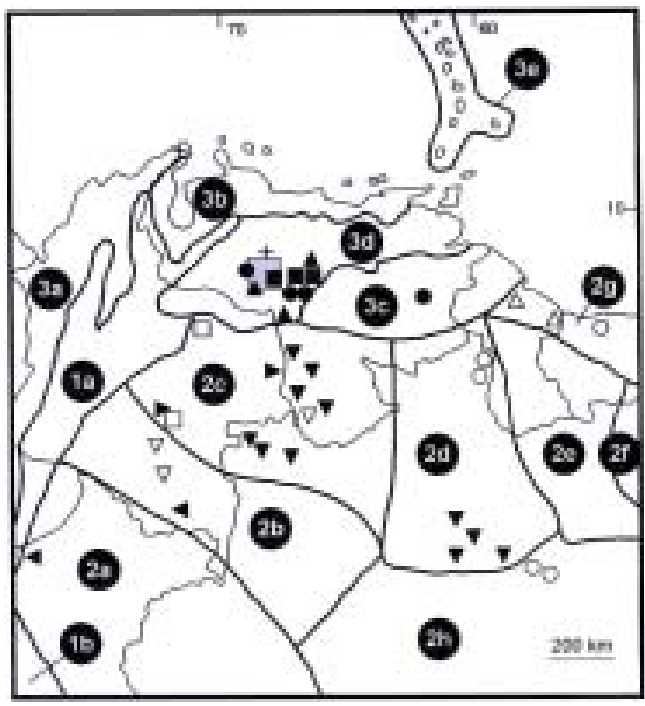

Figura 2. Sectorización biogeográfica del $\mathrm{N}$ de América del Sur. Biogeographical sectorization of northern South America: 1- Región Andina (subregión Paramuno-Puneña, superprovincia del Páramo), 2- Región Amazónica según las regiones de Prance (1977)(2a- Suroeste, 2bSolimões-Amazonas oeste, 2c- Noroeste Alto Río Negro, 2d- Roraima-Manaus, 2e- Jari-Trombetas, 2f y 2 g- Costa atlántica), 3- Región CaribeñoMesoamericana [3a- provincia Pacífica, 3bprovincia Costera-Venezolana, $3 \mathrm{c}$ - provincia de la Guayana, 3d- provincia de los Llanos (el cuadrado gris indica la ubicación del territorio estudiado), 3e-provincia del Caribe]. Símbolos:

- Randia venezuelensis, + Coccoloba obtusifolia, - Annona jahnii, - Duguetia riberensis, Rourea cuspidata, $\Delta$ Couratari gloriosa, $\mathbf{O}$ C. oblongifolia, $\square$ Pachira coriacea, Bonnetia martiana, 4 Dimorphandra cuprea, $\ominus$ Ilex divaricata.

Bursera tomentosa (Jacq.) Triana \& Planch., Casearia hirsuta Sw., Ceiba pentandra (L.) Gaertn., Genipa caruto Kunth, Guazuma ulmifolia Lam., Hamelia patens Jacq., Malvaviscus arboreus Cav., Samanea saman (Jacq.) Merr. y Spondias mombin L. (Loveless, 1960; Borhidi, 1991; SantiagoValentín \& Olmstead, 2004). Por tanto, el esquema biogeográfico de los Llanos del Orinoco queda de la siguiente forma:

\author{
Reino Neotropical \\ Subreino Caribeo-Amazónico \\ Región Caribeño-Mesoamericana \\ Provincia de los Llanos
}

\section{El territorio estudiado}

El territorio en el que hemos basado nuestro estudio está situado al O de San Fernando de Apure, entre las poblaciones de El Samán y Mantecal, en la zona del Hato El Frío $\left(7^{\circ} 50^{\circ} \mathrm{N}, 69^{\circ} \mathrm{O}\right)$, a una altitud media de 70-80 m (figs. 1 y 2). Se trata de una extensión de unas $78000 \mathrm{Ha}$ situada al S del río Apure, que vierte sus aguas al Orinoco.

A los ríos Apure y Orinoco desemboca un entramado de afluentes que recorren el piedemonte andino desde unos $200 \mathrm{~m}$ de altitud (Llanos de Barinas) y, hacia el E, forman una depresión que se conoce como llanos inundables (Llanos de Apure). Como es de suponer, los llanos inundables son muy ricos en flora y fauna ligadas a los medios acuáticos (Velasco \& Ayarzagüena, 1995).

Los Llanos son de origen cuaternario aluvial y las clases de suelos van a depender de la deposición de arcillas, limos y arenas. En el área de estudio nos encontramos con depresiones de suelos arcillosos, con los estratos superiores oscuros (esteros), áreas menos encharcadas de suelo limoso de color rojizo o amarillento (bajío), y suelos arenosos, casi siempre menos encharcados (banco)(Lasser, 1969; Sarmiento \& Monasterio, 1969). Estos materiales cuaternarios, de origen andino o guayanés, están depositados sobre una costra laterítica de origen terciario, más superficial hacia el E (Walter, 1997).

\section{Obtención de datos, nomenclatura}

Los datos de campo fueron tomados entre los años 2002 y 2005, siguiendo el método fitosociológico de Braun-Blanquet 
(1964), que permite comparar los inventarios fitosociológicos con los de otras áreas disyuntas. Las tablas fitosociológicas obtenidas fueron comparadas con otras disponibles en la literatura, procedentes de territorios con características más o menos similares de América del Sur y el Caribe, como Cuba (Borhidi, 1991), Paraguay (Galán de Mera \& Navarro, 1992), Brasil meridional (Bolòs et al., 1991), Antillas Menores (Foucault, 1981), Perú (Müller \& Gutte, 1985), Venezuela (Castroviejo \& López, 1985; Susach Campalans, 1989) y Bolivia (Haase, 1989, 1990).

La nomenclatura de los sintáxones sigue el Código Internacional de Nomenclatura Fitosociológica (Weber et al., 2000).

Aunque el conocimiento de la flora de Venezuela podemos decir que es elevado (Huber et al., 1998), la obra "Flora de Venezuela" está todavía incompleta. Por esta razón, la determinación y nomenclatura de los táxones tratados en el texto y las tablas están basados en varios trabajos (Aristeguieta, 1973; Velásquez, 1994; Steyermark et al., 1995-2005; Clark et al., 2000), la base de datos W3-TROPICOS (Missouri Botanical Garden) y algunas monografías (Steyermark, 1974; LópezPalacios, 1977; Gentry,1982).

Los pliegos de la flora estudiada para la realización del trabajo se conservan en el herbario USP.

\section{RESULTADOS Y DISCUSIÓN}

1. Bosques y arbustedas

(Clase Ceibetea occidentalis)

\section{Bromelio chrysanthae-Platymiscietum} pinnati Galán de Mera, González, Morales, Oltra \& Vicente Orellana ass. nova

[Tabla 2, holotypus ass. inv. 6]
Bosques semidecíduos umbríos ("mata") que alcanzan de 20 a $30 \mathrm{~m}$, donde sobresalen algunos árboles como Ceiba pentandra, Couroupita guianensis Aubl., Ficus guianensis Desv. ex Ham., Samanea saman, Platymiscium pinnatum, Pterocarpus rohrii Vahl o Spondias mombin. El sotobosque está formado por pequeñas palmeras [Bactris guineensis (L.) H.E. Moore, Desmoncus orthacanthus Mart.], grandes geófitos [Heliconia psittacorum L.f., Calathea latifolia (Willd. ex Link) Klotzsch, Dracontium polyphyllum L., Maranta arundinacea L.], gramíneas nemorales (Olyra latifolia L., Pharus latifolius L.), y es frecuente la Bromeliácea terrestre Bromelia chrysantha Jacq., indicadora de suelos arenosos en superficie con un período de inundación corto (Castillo, 1977). También son abundantes las lianas, que a veces aislan por fuera el bosque de las comunidades externas (Arrabidaea mollissima, Smilax cumanensis, Strychnos panamensis, Teramnus volubilis Sw.), y los epífitos en los estratos superiores [Polypodium polypodioides (L.) Watt, P. wagneri Mett., Tillandsia balbisiana Schult. f.]. La presencia casi constante de xerófitos con tallos trepadores, que indican la sequía durante algunos meses del año, le transmiten la originalidad fisionómica a este bosque, cuya asociación incluimos dentro de DesmoncoPlatymiscion pinnati Galán de Mera, González, Morales, Oltra \& Vicente Orellana all. nova (holotypus: Bromelio chrysanthaePlatymiscietum pinnati Galán de Mera, González, Morales, Oltra \& Vicente Orellana ass. nova).

- copernicietosum tectorum Galán de Mera, González, Morales, Oltra \& Vicente Orellana subass. nova (holotypus: inv. 8)

Hasta el momento se habían descrito cuatro asociaciones con palmeras propias de las áreas neotropicales que se inundan (Rangel et al., 1997; Galán de Mera, 2001a; 
Tabla 2

\section{Bromelio chrysanthae-Platymiscietum pinnati ass.nova \\ (Ceibetea occidentalis, Bactrido-Cecropietalia, Desmonco-Platymiscion)}

$\begin{array}{lcccccccccccccc}\text { Inventario } \mathrm{n}^{\circ} & 1 & 2 & 3 & 4 & 5 & 6 & 7 & 8 & 9 & 10 & 11 & 12 & 13 & 14 \\ \text { Área }\left(\mathrm{m}^{2}\right) & 100 & 100 & 100 & 200 & 100 & 100 & 100 & 100 & 100 & 100 & 100 & 100 & 100 & 100\end{array}$

Características de asociación y alianza

Platymiscium pinnatum

Desmoncus orthacanthos

Olyra latifolia

Bromelia chrysantha

Pithecellobium ligustrinum

Pterocarpus rohrii

Couroupita guianensis

$\begin{array}{llllllllllllll}. & . & 1 & 1 & 4 & 3 & 4 & 4 & 2 & . & . & . & 3 & 3 \\ . & . & + & 1 & . & . & . & + & . & . & . & . & 1 & . \\ . & 1 & 1 & . & . & . & . & . & . & . & 1 & . & . & . \\ . & . & . & . & + & 1 & . & 1 & . & . & . & . & . & . \\ . & . & . & . & . & 1 & . & . & + & . & . & . & . & . \\ . & 4 & . & . & 1 & . & . & . & . & . & . & . & . & . \\ 3 & . & . & . & . & . & . & . & . & . & . & . & . & . \\ \end{array}$

diferenciales de subasociaciones

Hura crepitans

Copernicia tectorum

Calyptranthes pullei

Duguetia riberensis

Calathea latifolia

Nectandra pichurim

Adenocalymna apurense

Características de orden

Bursera tomentosa

Coccoloba caracasana

Cecropia peltata

Bactris guineensis

Genipa caruto

Psychotria microdon

Dracontium polyphyllum

Características de clase

Strychnos panamensis

Teramnus volubilis

Lithachne pauciflora

Guazuma ulmifolia

Samanea saman

Psychotria anceps

Hamelia patens

Malvaviscus arboreus

Palicourea crocea

Cochlospermum vitifolium

Maranta arundinacea

Smilax cumanensis

Spondias mombin

Piper tuberculatum

Arrabidaea mollissima

Heliconia psittacorum

Doliocarpus dentatus

Ficus guianensis

Margaritaria nobilis

Diospyros guianensis

Ceiba pentandra

Bauhinia glabra

Pithecelobium pistaciaefolium 


\begin{tabular}{|c|c|c|c|c|c|c|c|c|c|c|c|c|c|c|}
\hline Inventario $\mathrm{n}^{\mathrm{o}}$ & 1 & 2 & 3 & 4 & 5 & 6 & 7 & 8 & 9 & 10 & 11 & 12 & 13 & 14 \\
\hline Lantana moritziana & . & . & & . & + & . & . & . & . & . & . & . & 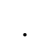 & \\
\hline Caesalpinia coriaria & . & . & 1 & & . & . & . & . & . & . & . & . & . & \\
\hline Seguieria aculeata & . & . & . & 1 & . & . & . & . & . & . & . & & . & \\
\hline Cordia collococca & . & . & . & . & . & . & . & . & . & . & . & 1 & . & \\
\hline Randia venezuelensis & . & . & . & . & . & . & . & . & . & . & . & . & . & + \\
\hline Annona jahnii & . & . & . & . & 1 & . & . & . & . & . & . & . & . & \\
\hline Entada polystachya & . & . & . & . & 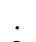 & . & . & . & . & 1 & . & . & . & \\
\hline Ipomoea trifida & . & . & . & . & 2 & . & . & . & . & . & . & . & . & \\
\hline Bauhinia benthamiana & . & . & + & . & . & . & . & . & . & : & . & . & . & \\
\hline Gouania polygama & . & . & . & . & . & . & . & . & . & 1 & . & . & . & \\
\hline Byrsonima crassifolia & . & . & . & . & . & . & . & . & . & 1 & . & . & . & \\
\hline Zanthoxylum fagara & . & . & . & . & . & . & . & . & . & 2 & . & . & . & \\
\hline \multicolumn{15}{|l|}{ Compañeras } \\
\hline Solanum sp. & . & 1 & . & & . & . & . & . & . & i & + & 1 & . & + \\
\hline Phthrirusa stelis & 1 & + & . & 1 & . & - & . & . & . & 1 & & . & & \\
\hline Phylodendron scandens & . & . & . & 1 & . & + & . & . & $\cdot$ & . & 1 & . & 1 & \\
\hline Mimosa pudica & . & . & . & . & . & . & . & . & + & . & . & . & . & \\
\hline Mimosa schrankioides & . & + & . & . & . & . & . & . & . & . & . & . & & \\
\hline Momordica charantia & . & . & . & . & . & . & $\dot{0}$ & . & . & . & . & . & 1 & + \\
\hline Manguifera indica & + & . & . & . & . & . & 2 & . & . & . & . & . & . & \\
\hline
\end{tabular}

Otras plantas: Phyllanthus acidus 1 en 1; Pipthadenia obliqua 1 en 2; Amaranthus spinosus + en 5; Aeschynomene sp.1 en 8; Mimosa pudica +, Melastomataceae + en 9; Proteaceae 1, Mimosa sp.+, Caraipa sp.1 en 12; Crescentia cujete +, Euphorbia hyssopifolia 1 en 13.

Localidades: 1-5, 7: La Carmera; 6, 8, 9: Mata Silva; 10, 11, 13: Caño Guaritico; 12, 14: Río Apure.

Galán de Mera, 2001b): Ilico laureolaeMauritietum flexuosae Urrego 1994 (palmerales del S de Colombia), Oenocarpo maporae-Mauritietum flexuosae Galán de Mera 1996 (aguajales de la Cuenca Amazónica peruana), Pachiro brevipedisEuterpetum catingae Galán de Mera 2001 (varillales húmedos de la Cuenca Amazónica peruana) y Prosopido vinalillonisCopernicietum albae Galán de Mera 2001 (palmerales chaqueños). En Venezuela podemos distinguir los "morichales llaneros" con Mauritia flexuosa L.f., próximos a los grandes ríos (Aristeguieta, 1968a), y los "palmerales llaneros" con Copernicia tectorum (H.B.K.) Mart., característicos de suelos con inundación estacional (Hoyos F. y Braun, s.f.).

En nuestro territorio, los bosques con Copernicia tectorum muestran inundación durante varios meses al año y se asientan sobre suelos impermeables, poco oxigenados. En los Llanos, C. tectorum, al igual que Acrocomia aculeata (Jacq.) Lodd. ex Mart., puede aparecer de forma más o menos dispersa formando mosaico con pastizales. Posiblemente esto sea debido al desmonte y quemado de los bosques favoreciendo a los pastos, y a la resistencia de las palmeras al fuego, aunque en realidad su posición natural es formar bosques (Lasser, 1969; Comerma y Luque, 1971). Entre las características de esta subasociación también destacamos a Hura crepitans L., que como en los bosques inundables de fisiografía plana de la Amazonía, es una especie importante (Encarnación, 1985).

- duguetietosum riberensis Galán de Mera, González, Morales, Oltra \& Vicente Orellana subass. nova (holotypus: inv. 14) (Sin.: Nectandro pichurini-Duguetietum 
Tabla 3

Randio venezuelensis-Annonetum jahnii ass. nova

(Ceibetea occidentalis, Bactrido-Cecropietalia, Nectandro-Viticion)

Inventario $\mathrm{n}^{\mathrm{o}}$

Área $\left(\mathrm{m}^{2}\right)$

$\begin{array}{cccccccccccccccccc}1 & 2 & 3 & 4 & 5 & 6 & 7 & 8 & 9 & 10 & 11 & 12 & 13 & 14 & 15 & 16 & 17 & 18 \\ 1 & 1 & 1 & 1 & 1 & 1 & 1 & 1 & & 1 & & & & 1 & & & & 1 \\ 0 & 0 & 0 & 0 & 0 & 0 & 0 & 0 & 5 & 0 & 5 & 5 & 2 & 0 & 3 & 2 & 5 & 0 \\ 0 & 0 & 0 & 0 & 0 & 0 & 0 & 0 & 0 & 0 & 0 & 0 & 0 & 0 & 0 & 0 & 0 & 0\end{array}$

Características de asociación y alianza

Randia venezuelensis

Annona jahnii

Vitex orinocensis

Nectandra globosa

Cordia hirta

Diospyros guianensis

Passiflora pulchella

Sapium glandulosum

Copaifera officinalis

Casearia hirsuta

Lianas y arbustos escandentes

Passiflora foetida

Cissus sicyoides

Passiflora guazumifolia

Ipomoea trifida

Arrabidaea corallina

Cissus elata

Entada polystachya

Cissampelos pareira

Características de orden y clase

Guazuma ulmifolia

Arrabidaea mollissima

Malvaviscus arboreus

Palicourea crocea

Gouania polygama

Byrsonima crassifolia

Zanthoxylum fagara

Samanea saman

Bursera tomentosa

Coccoloba caracasana

Psychotria microdon

Ceiba pentandra

Bactris guineensis

Genipa caruto

Lithachne pauciflora

Strychnos panamensis

Psychotria anceps

Smilax cumanensis

Spondias mombin

Doliocarpus dentatus

Cardiospermum halicacabum .

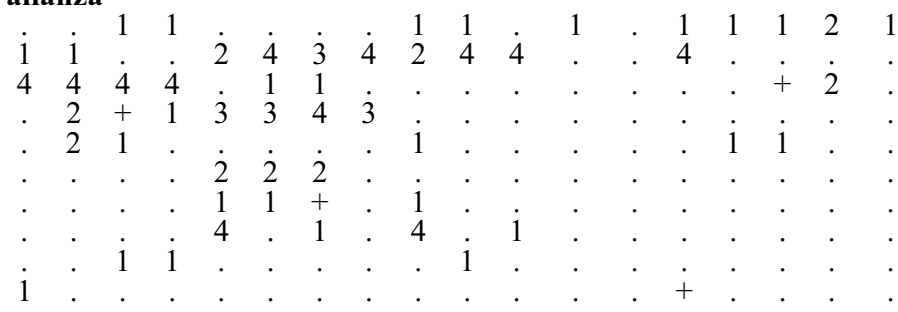

Pithecelobium pistaciaefolium

Bauhinia glabra

Compañeras

Mimosa pudica

Solanum sp.

Phthrirusa stelis

Mimosa schrankioides

Solanum mammosum

Otras plantas: Combretum laxum 1 en 1; Sarcostemma clausum 1 en 5; Ipomoea asarifolia + en 7; Aniseia martinicensis 1, Indigofera suffruticosa + , Calopogonium mucunoides 1, Hystis sp. + en 8; Humiria balsamifera + en 10; Cleome spinosa + en 12; Momordica charantia 1 en 13; Eupatorium odoratum 1 en 14. Localities: 1, 2, 11-15, 16-18-Caño Mucuritas; 3, 4-Caño Macanillal; 5-10- estación biológica; 8-Matagorda. 
riberensis Castroviejo \& López 1985, prov.)

El bosque de Bromelio-Platymiscietum a veces incluye Duguetia riberensis, un árbol indicador de aguas corrientes que registran crecidas estacionales originando un bosque de galería.

A diferencia de Castroviejo \& López (1985), pensamos que se trata de una subasociación de bosque de mata puesto que en su interior siguen apareciendo elementos como Bursera tomentosa, Coccoloba caracasana Meisn. o Desmoncus orthacanthos.

Los bosques de los Llanos Occidentales contienen plantas de areal muy extenso, y plantas que se distribuyen por el $\mathrm{N}$ de América del Sur, Centroamérica y el Caribe, mostrándose vicariantes del orden OxandroBurseretalia Borhidi \& Muñiz in Borhidi 1996, descrito con asociaciones de Cuba (Borhidi, 1996)[características: Amyris balsamifera L., A. elemifera L., Andira inermis (W. Wright) Kunth ex DC., Buchenavia capitata (Vahl) Eichl., Bursera simaruba (L.) Sarg., Casearia hirsuta, $C$. spinescens (Sw.) Griseb., Cordia collococca L., C. gerascanthus L., Hypelate trifolia Sw., Jacaranda caerulea (L.) J. St.-Hil., Pithecellobium cubense Bisse, Samanea saman, Spondias mombin, Zanthoxylum elephantiasis Macfad.]. Por tanto, podemos describir el nuevo orden BactridoCecropietalia Galán de Mera, González, Morales, Oltra \& Vicente Orellana ord. novo (holotypus: Desmonco-Platymiscion pinnati Galán de Mera, González, Morales, Oltra \& Vicente Orellana all. nova).

Además, los bosques en nuestra zona de estudio soportan diferentes grados de inundación durante la época de lluvias. Esto conlleva la ausencia de ciertas especies [Albizia caribaea (Urb.) Britton \& Rose, Astronium graveolens Jacq., Bombacopsis quinata (Jacq.) Dugand, Sorocea sprucei
(Baill.) J.F. Macbr., Swietenia macrophylla King] presentes en los bosques situados más al norte, con una mayor altitud (Cuello et al., 1989; Plonczak, 1998). Por contra, también una buena cantidad de especies están en otros bosques húmedos de América del Sur; por ejemplo, Ceiba pentandra, Guazuma ulmifolia, Palicourea crocea (Sw.) Roem. \& Schult., Psychotria anceps Kunth o Spondias mombin, también están en los bosques húmedos de San Carlos de Río Negro (Venezuela)(Clark et al., 2000), en la Amazonía peruana (Vásquez Martínez, 1997) o en las selvas del Beni (Bolivia)(Killeen et al., 1993), por lo que parece aconsejable reconocer la clase Ceibetea occidentalis Knapp ex Borhidi 1996, descrita de América Central y el Caribe (Knapp, 1964).

\section{Randio venezuelensis-Annonetum jahnii}

Galán de Mera, González, Morales, Oltra \& Vicente Orellana ass. nova

[Tabla 3, holotypus ass. inv. 9]

Vegetación de unos $6 \mathrm{~m}$ de altura, que se asienta sobre suelos limo-arenosos. Se caracteriza por la presencia de Randia venezuelensis y Annona jahnii, dos endemismos venezolanos (Steyermark, 1974; Steyermark et al., 1995). La presencia frecuente de lianas [Cissampelos pareira, Ipomoea trifida (H.B.K.) G. Don, Passiflora foetida L.] indica los aspectos más húmedos de la asociación.

\section{Helictero guazumifoliae-Bauhinietum benthamianae Galán de Mera, González, Morales, Oltra \& Vicente Orellana ass. nova \\ [Tabla 4, holotypus ass. inv. 4]}

Los suelos con arcillas amarillentas, sin arenas, soportan esta comunidad de árboles $\mathrm{y}$ arbustos que llegan a alcanzar unos $5 \mathrm{~m}$. 
Tabla 4

Helictero guazumifoliae-Bauhinietum benthamianae ass. nova

(Ceibetea occidentalis, Bactrido-Cecropietalia, Nectandro-Viticion)

Inventario $\mathrm{n}^{\mathrm{o}}$

Área $\left(\mathrm{m}^{2}\right)$

$\begin{array}{cccccc}1 & 2 & 3 & 4 & 5 & 6 \\ 100 & 50 & 50 & 100 & 100 & 50\end{array}$

Características de asociación y alianza

Bauhinia benthamiana

Helicteres guazumifolia

Lygodium venustum

Vitex orinocensis

Nectandra globosa

Randia venezuelensis

Annona jahnii

Características de orden y clase

Guazuma ulmifolia

Cecropia peltata

Malvaviscus arboreus

Teramnus volubilis

Coccoloba caracasana

Bactris guineensis

Arrabidaea mollissima

Samanea saman

Lithachne pauciflora

Byrsonima crassifolia

Zanthoxylum fagara

Hamelia patens

Cochlospermum vitifolium

Cardiospermum halicacabum

Pithecellobium ligustrinum

Cissus sicyoides

Cissampelos pareira

Compañeras

Phthrirusa stelis

Solanum sp.

Mimosa pudica

Momordica charantia

Otras plantas: Solanum mammosum + , Mikania micrantha 1 en 1; Psittacanthus calyculatus + en 1 . Localidades: 1-6- La Carmera.

En ocasiones esta asociación muestra un aspecto alterado donde Bauhinia benthamiana Taub. se comporta como pionera por abandono de los pastizales sabaneros inundables que han sido quemados.

Si la alianza Desmonco-Platymiscion reúne a elementos de bosques húmedos, la alianza Nectandro-Viticion Galán de Mera, González, Morales, Oltra \& Vicente Orellana all. nova (holotypus: Randio venezuelensisAnnonetum jahnii Galán de Mera, González, Morales, Oltra \& Vicente Orellana ass. nova) se caracteriza por plantas heliófilas donde la esclerofilia se hace más evidente como consecuencia de una mayor evapotranspiración. Las arbustedas pueden pioneras o situarse fitotopográficamente entre las matas y los pastizales inundables. Por ello son refugio de animales que 
Tabla 5

1-6: Cassio rotundifoliae-Elionuretum tripsacoidis Castroviejo \& López 1985

7-8: Panico juncei-Imperatetum contractae Castroviejo \& López 1985

(Leptocoryphio-Trachypogonetea, Trachypogonetalia, Elyonurion)

Inventario $\mathrm{n}^{\circ}$

Área $\left(\mathrm{m}^{2}\right)$

Número de inventarios

Características de Cassio-Elionuretum

Cassia rotundifolia

Brachiaria fasciculata

Mimosa quadrivalvis var. leptocarpa

Borreria aristeguietana

Oxalis barrelieri

Eleusine indica

Características de Panico-Imperatetum

Pavonia sessiliflora

Scleria hirtella

Imperata contracta

Panicum junceum

Borreria capitata

Sida linifolia

Crotalaria pilosa

Hyptis conferta var. angustata

Crotalaria incana

Características de alianza

Calopogonium mucunoides

Elionurus tripsacoides

Sida glomerata

Cyperus flavus

Cyperus sphacelatus

Macroptilium lathyroides

Euphorbia hirta

Paspalum plicatulum

Setaria geniculata

Cyperus odoratus

Fymbristylis dichotoma

Cyperus aggregatus

Cyperus aggregatus
Características de orden y clase

Axonopus purpusii

Dichromena ciliata

Cyperus luzulae

Desmodium scorpiurus

Eragrostis maypurensis

Sporobolus indicus

Kyllinga brevifolia

Cyperus surinamensis

Compañeras

Kyllinga odorata

Desmodium barbatum

Turnera ulmifolia

Sida rhombifolia

Ludwigia hyssopifolia

Murdannia nudiflora

Melastomataceae

Marsypianthes sp.

Cuphea elliptica

Melochia parvifolia

$\begin{array}{cccccccc}1 & 2 & 3 & 4 & 5 & \underline{6} & 7 & \underline{8} \\ 100 & 100 & 100 & 100 & 200 & . & 50 & . \\ . & . & . & . & . & 4 & . & 6\end{array}$

V

V

V

V

II

I

$\begin{array}{llllllll}. & 2 & 1 & 1 & 1 & 4 & 2 & \mathrm{~V} \\ 2 & \cdot & \mathrm{i} & \cdot & 2 & 4 & + & \mathrm{V} \\ & . & & & & & & \end{array}$

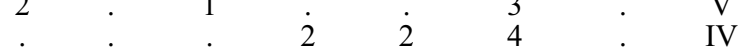

$\begin{array}{rllllll}. & 1 & . & 1 & 4 & \text {. } & \text { II }\end{array}$

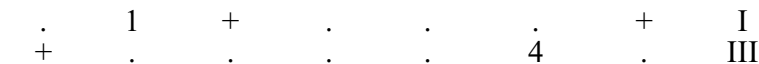

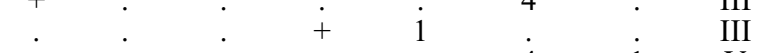

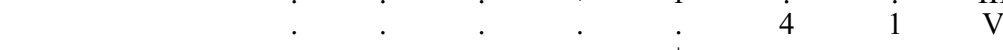




\begin{tabular}{|c|c|c|c|c|c|c|c|c|}
\hline Inventario $\mathrm{n}^{\mathrm{o}}$ & 1 & 2 & 3 & 4 & 5 & $\underline{6}$ & 7 & $\underline{8}$ \\
\hline Hyptis suaveolens & . & . & . & . & . & 3 & . & III \\
\hline Cassia tora & . & . & . & . & . & 3 & . & III \\
\hline Pectis elongata & . & . & . & . & . & 2 & . & III \\
\hline Sida acuta & . & . & . & . & . & 1 & . & I \\
\hline Stylosanthes humilis & . & . & . & . & . & 2 & . & II \\
\hline Euphorbia hyssopifolia & . & . & . & . & . & & + & III \\
\hline Convolvulaceae & . & . & . & . & . & 2 & . & II \\
\hline Indigofera pascuorum & . & . & . & . & . & 1 & . & III \\
\hline Elephantopus mollis & . & . & . & . & . & 3 & . & I \\
\hline Paspalum sp. & . & . & . & . & & 1 & . & I \\
\hline Hyptis mutabilis & . & . & & 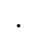 & 1 & & . & III \\
\hline Diodia teres var. prostrata & . & . & 1 & . & . & 2 & . & \\
\hline Panicum versicolor & . & . & & & & 2 & . & I \\
\hline Cuphea brevipes & . & . & 2 & 1 & & . & . & $\cdot$ \\
\hline Cyperus tenuis & . & + & & & 1 & . & . & . \\
\hline Solanum sessiliflorum & . & . & 1 & + & . & . & . & \\
\hline Rhynchospora sp. & . & . & . & . & . & 1 & . & I \\
\hline Scoparia dulcis & . & . & . & . & . & 1 & . & I \\
\hline
\end{tabular}

Otras plantas: Rhynchospora capitata 1 en 1; Hyptis sedioides 2 en 2; Cyperus laxus 3 en 3; Callisia filiformis 1, Momordica charantia 1, Amaranthus spinosus 2, Cassia aculeata + , Sida procumbens + en 4; Ludwigia inclinata + en 5; Cenchrus pilosus 3, Ipomoea sp. 1, Borreria ocymoides 1, Bidens aff. pilosa 1 en 6 ; Urena sinuata II, Sebastiana corniculata I, Peperomia sp. I, Phaseolus sp. I, Borreria latifolia I, Evolvulus nummularius I, Caperonia palustris I, Fimbristylis sp. I, Borreria verticillata I, Cassia calycioides IV en $\underline{8}$.

Localidades: 1- estación biológica, 2- camino del Caño Mucuritas, 3- de la estación biológica a la mata La Ramera; 4- de la estación biológica al aeropuerto; 5- aeropuerto, $\underline{6}$ y $\underline{8}$ - algunas localidades en el Hato El Frío (Castroviejo \& López, 1985); 7- Matagorda.

dependen en gran parte del agua, como la capibara o chigüire (Hydrochoeris hydrochoeris L.), o el cocodrilo del Orinoco (Crocodylus intermedius Graves) que utiliza los suelos arenosos para desovar.

\section{Las arbustedas ribereñas (Clase Coccolobietea obtusifoliae)}

Coccolobetum obtusifoliae Castroviejo \& López 1985

Las comunidades con Coccoloba uvifera (L.) L. están bastante extendidas por las costas arenosas del Caribe (Borhidi et al., 1979, Knapp, 1980). Coccoloba obtusifolia también tiene preferencias por suelos con una elevada proporción de arenas (Castillo, 1977). Se trata de arbustedas de 2-4 m de altura que se desarrollan sobre los materiales que depositan los ríos de gran caudal; es decir, sobre todo en la cara interna de los meandros y en los tramos rectos, donde Coccoloba obtusifolia, con sus raíces adventicias, soporta una constante inundación con grandes oscilaciones. Por tanto, los "manglares" (en sentido local) de Coccoloba están en contacto sobre todo con los pastizales flotantes de Hymenachnion amplexicaulis, que abordaremos más adelante.

Coccolobetum obtusifoliae es una asociación que sólo conocemos de los Llanos del Orinoco y del lago de Maracaibo. Al igual que ocurre con los manglares de agua salada, es una asociación con una combinación florística bastante pobre (Peinado et al., 1995) que nos lleva a describir para esta única asociación, la alianza Coccolobion obtusifoliae Galán de Mera, González, Morales, Oltra \& Vicente Orellana all. nova (holotypus: Coccolobetum obtusifoliae 
Castroviejo \& López 1985), el orden Coccolobietalia obtusifoliae Galán de Mera, González, Morales, Oltra \& Vicente Orellana ordo novo (holotypus: Coccolobion obtusifoliae Galán de Mera, González, Morales, Oltra \& Vicente Orellana all. nova) y la clase Coccolobietea obtusifoliae Galán de Mera, González, Morales, Oltra \& Vicente Orellana cl. nova (holotypus: Coccolobietalia obtusifoliae Galán de Mera, González, Morales, Oltra \& Vicente Orellana ordo novo).

\section{Los pastizales \\ (Clase Leptocoryphio-Trachypogonetea)}

Los pastizales llaneros fueron ampliamente estudiados por Castroviejo \& López (1985) en los llanos occidentales, Susach Campalans (1989) en la parte más occidental de los llanos orientales, y Van Donselaar (1965) en las sabanas de Surinam.

\section{Cassio rotundifoliae-Elionuretum} tripsacoidis Castroviejo \& López 1985 [Tabla 5]

Son los pastizales de las áreas arenosas no inundables de los llanos occidentales (bancos). Están constituidos sobre todo por gramíneas perennes que alcanzan $1 \mathrm{~m}$ de altura cuando están bien desarrollados, y un sustrato de leguminosas y oxalidáceas (Calopogonium mucunoides Desv., Oxalis barrelieri L.) que aportan palatabilidad. Son además ricos en Cyperáceas, como Cyperus luzulae (L.) Rottb. ex Retz., C. sphacelatus Rottb., Dichromena ciliata Vahl o Kyllinga brevifolia Rottb., y debido al sobrepastoreo al que se ven sometidos en la época de lluvias, se introducen numerosas plantas nitrófilas (Amaranthus spinosus L., Hyptis brevipes Poit., Momordica charantia L., Sida procumbens Sw., S. rhombifolia L.) e incluso algunas especies alóctonas de origen paleotropical [Eleusine indica (L.) Gaertn., Macroptilium lathyroides (L.) Urb.].

El abandono del pastizal conduce a la aparición de Mimosa quadrivalvis var. leptocarpa, especie que puede considerarse pionera de las comunidades de NectandroViticion.

De acuerdo con Castroviejo \& López (1985) incluimos esta asociación en la alianza Elionurion tripsacoidis y, aunque no hemos encontrado en el territorio Trachypogon plumosus (Humb. \& Bonpl. ex Willd.) Nees, la presencia de Axonopus purpusii (Mez) Chase, Dichromena ciliata y Eragrostis maypurensis (Kunth) Steud., nos llevan a considerar el orden Trachypogonetalia plumosi y la clase Leptocoryphio-Trachypogonetea (Susach Campalans, 1989). Los pastizales de Trachypogon plumosus y Leptocoryphium lanatum (Kunth) Nees también forman parte de las sabanas del N de Bolivia (Haase, 1990), aunque con una composición florística diferente [p.ej. Andropogon leucostachyus Kunth, Mesosetum penicillatum Mez, Paspalum ionanthum Chase, Thrasya petrosa (Trin.) Chase].

\section{Panico juncei-Imperatetum contractae}

Castroviejo \& López 1985

[Tabla 5]

Pastizales de unos $50 \mathrm{~cm}$ de altura característicos de suelos arenosos que soportan algo de inundación durante la época de lluvias. Están dominados por Imperata contracta (Kunth) Hitchc. y, al igual que la asociación anterior, presentan un sustrato de leguminosas representado sobre todo por Calopogonium mucunoides.

El aumento de la humedad edáfica permite el contacto con los pastos de Eleocharis minima, aumentando las especies propias de medios húmedos [Caperonia palustris (L.) A. St.-Hil., Ludwigia hyssopifolia (G. Don) Exell]. 
Tabla 6

Spilantho uliginosi-Paspaletum orbiculati Castroviejo \& López 1985 (Xyridetea, Eleocharitetalia minimae, Oryzopsion perennis)

\begin{tabular}{|c|c|c|c|c|c|c|}
\hline Inventario $\mathrm{n}^{\mathrm{o}}$ & 1 & 2 & 3 & 4 & $\underline{5}$ & $\underline{6}$ \\
\hline Área $\left(\mathrm{m}^{2}\right)$ & 4 & 4 & 2 & 5 & & \\
\hline Número de inventarios & . & . & . & . & 9 & 3 \\
\hline \multicolumn{7}{|c|}{ Características de asociación y unidades superiores } \\
\hline Eleocharis minima & 4 & 3 & 5 & 4 & IV & 3 \\
\hline Paspalum orbiculatum & 4 & 3 & 5 & 4 & IV & . \\
\hline Oryza perennis & 2 & & 1 & 2 & I & . \\
\hline Bacopa monnierioides & 3 & 2 & 2 & & III & . \\
\hline Lindernia crustacea & 1 & . & . & 2 & II & . \\
\hline Cyperus flavescens & . & . & . & + & III & . \\
\hline Leersia hexandra & . & . & . & 2 & I & . \\
\hline Luziola pittieri & . & . & . & . & IV & . \\
\hline Bacopa salzmanii & . & . & . & . & IV & . \\
\hline Panicum laxum & . & . & . & . & IV & . \\
\hline Spilanthes uliginosa & . & . & . & . & IV & . \\
\hline Borreria cf. aristeguietana & . & . & . & . & IV & . \\
\hline Acisanthera quadrata & . & . & . & $v^{\circ}$ & III & . \\
\hline \multicolumn{7}{|l|}{ Compañeras } \\
\hline Caperonia palustris & + & . & . & + & IV & \\
\hline Ludwigia hyssopifolia & & & . & + & $\mathrm{V}$ & . \\
\hline Ipomoea asarifolia & + & & $\cdot$ & . & III & \\
\hline Ludwigia inclinata & . & 1 & . & . & . & 1 \\
\hline Sporobolus indicus & . & 2 & + & . & & r. \\
\hline
\end{tabular}

Otras plantas: Conobea aquatica + , Luziola spruceana 1, Hymenachne amplexicaulis 1 , Heteranthera limosa + en 1; Borreria aristeguietana + , Hibiscus furcellatus 1, Ipomoea fistulosa,+ Limnocharis flava +, Diodia teres var. prostrara +, Cuphea brevipes 1, Rhynchospora capitata 1, Sida glomerata + en 4; Philodice hoffmannseggii + , Sagittaria guyanensis,+ Eclipta alba + , Limnocharis sp. 1, Hyptis pulegioides +, Cyperus surinamensis I, Alternanthera crucis II, Rhynchospora sp. I, Kyllinga cf. brevifolia II, Cyperaceae I, Phyllanthus orbiculatus II, Eragrostis acutiflora III, Sacciolepis myuros III, Melochia pilosa III, Poaceae III, Fimbristylis aff. dichotoma III, Soemmeringia semperflorens III, Paspalum sp. II, Stylosanthes humilis I, Sida acuta II, Oldenlandia lancifolia III, Fimbristylis miliacea III, Aeschynomene evenia III, Ludwigia sp. III, Pectis elongata I, Borreria verticillata IV, Kyllinga odorata II, Murdannia nudiflora IV en $\underline{5}$; Eleocharis sp. 1, Hydrolea spinosa + , Ludwigia sedioides + , Marsilea deflexa + en $\underline{6}$. Localidades: 1-3-camino del Caño Mucuritas, 4- entrada del Hato El Frío, $\underline{5}$ y $\underline{6}$ - algunas localidades en el Hato El Frío (Castroviejo \& López, 1985).

La presencia constante de Calopogonium mucunoides, Elionurus tripsacoides Humb. \& Bonpl. ex Willd., Setaria geniculata P. Beauv. y Sida glomerata Cav. no ofrecen dudas sobre la inclusión de esta asociación en la alianza Elionurion tripsacoidis.

\section{Spilantho uliginosi-Paspaletum orbiculati}

Castroviejo \& López 1985

[Tabla 6]
Esta asociación son los bajíos con Eleocharis minima Kunth de los Llanos del Orinoco. Se trata de un pastizal de suelos encharcados durante la época de lluvias, donde además adquieren gran importancia las plantas anuales. Las características más distintivas de esta comunidad son Bacopa monnierioides (Cham.) B.L. Rob., Eleocharis minima, Lindernia crustacea (L.) F. Muell. y Paspalum orbiculatum Poir., formando una situación intermedia entre 


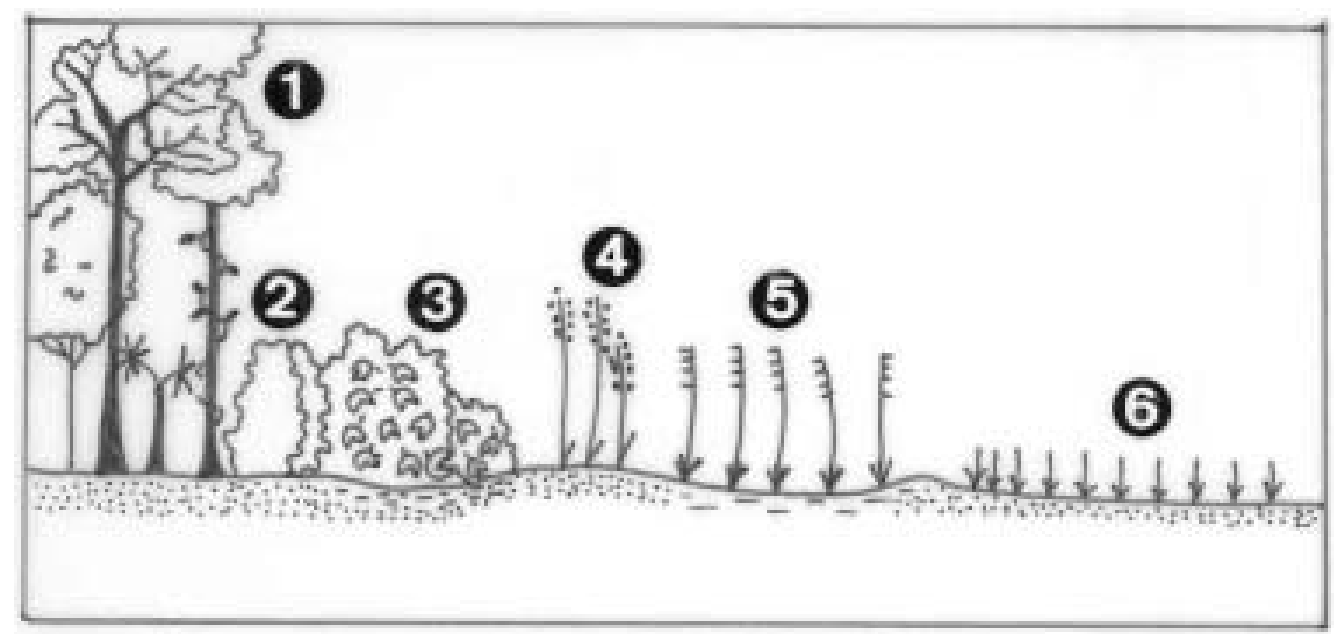

Figura 3. Esquema de los bosques y los pastizales en el territorio estudiado. Scheme of the forest and the grasslands in the studied territory. 1- Bromelio chrysanthae-Platymiscietum pinnati, 2-Randio venezuelensis-Annonetum jahnii, 3- Helictero guazumifoliae-Bauhinietum benthamianae, 4- Cassio rotundifoliae-Elionuretum tripsacoidis, 5- Panico juncei-Imperatetum contractae, 6- Spilantho uliginosi-Paspaletum orbiculati

Panico juncei-Imperatetum contractae y los juncales de Eleocharis mutata (L.) Roem. \& Schult., que prefieren suelos permanentemente encharcados (figs. 3 y 4).

La presencia casi constante de Oryza perennis Moench, especie de Centroamérica y $\mathrm{N}$ de América del Sur, nos lleva a crear la alianza Oryzion perennis Galán de Mera, González, Morales, Oltra \& Vicente Orellana all. nova (holotypus: Spilantho uliginosiPaspaletum orbiculati Castroviejo \& López 1985) y el orden Eleocharitetalia minimae Galán de Mera, González, Morales, Oltra \& Vicente Orellana ordo novo (holotypus: Oryzion perennis Galán de Mera, González, Morales, Oltra \& Vicente Orellana all. nova), que diferencia a las comunidades con Eleocharis minima de las de PaepalanthoEriocauletalia Knapp ex Bal.-Tul. \& Capote 1985 y Rhynchosporo-Xyridetalia Borhidi in Bal.-Tul. \& Capote 1985 de las Antillas (Borhidi, 1996). También incluimos en este nuevo orden a las comunidades con Eleocharis minima del norte de Bolivia
(Haase, 1989). Especies como Bacopa monnierioides, B. salzmanii (Benth.) Wettst. ex Edwall o Lindernia crustacea permiten la inclusión de estos pastizales en la clase Xyridetea savanensis (Galán de Mera, 1995).

\section{Las comunidades helofíticas \\ (Clase Cladietea jamaicensis)}

Eleocharitetum mutatae Castroviejo \& López 1985

[Tabla 7]

Juncal de unos $30 \mathrm{~cm}$ de alto, de aspecto verde brillante, que se muestra en contacto con la asociación Spilantho uliginosiPaspaletum orbiculati situándose en áreas con encharcamientos de 20-40 cm. Esto hace que en época de lluvias a veces se presente formando un mosaico con Marsilea deflexa A. Braun, que se comporta en el territorio como el hidrófito de aguas más someras. Es una asociación bastante pobre en especies por lo que no hemos podido observar 


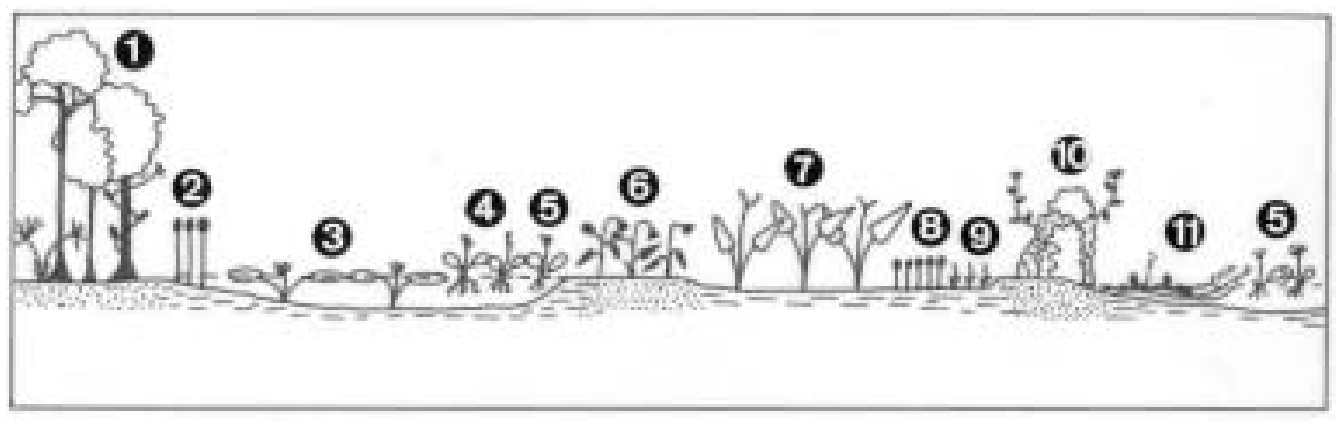

Figura 4. Esquema de las comunidades helofíticas y acuáticas en el territorio estudiado. Scheme of the helophytic and aquatic communities in the studied territory. 1-Bromelio chrysanthaePlatymiscietum pinnati, 2- Eleocharitetum interstinctae, 3- Ludwigio sedioidis-Eichhornietum diversifoliae, 4- Eichhornietum azureae, 5-Eichhornietum crassipedis, 6- Comunidad de Ipomoea carnea subsp. fistulosa, 7- Thalietum geniculatae, 8-Eleocharitetum mutatae, 9- Spilantho uliginosiPaspaletum orbiculati, 10- Arbusteda pionera de Randio venezuelensis-Annonetum jahnii (variante húmeda con lianas), 11- Luziolo-Hymenachnetum amplexicaulis.

variabilidad entre diferentes regiones geográficas (Foucault, 1981).

Eleocharitetum interstinctae Castroviejo \& López 1985

[Tabla 7]

Es el juncal de medios más inundados, hasta $100 \mathrm{~cm}$ de profundidad. Aparece en los esteros donde se acumula una mayor cantidad de lodo, y destaca como una comunidad de aspecto glauco cuyas características suelen ir acompañadas de elementos acuáticos [Eichhornia crassipes (Mart.) Solms, Hymenachne amplexicaulis (Rudge) Nees, Salvinia auriculata Aubl.].

La ausencia de algunas plantas presentes en las Antillas [Cyperus ligularis L., Pontederia lanceolata Nutt., Rhynchospora colorata (L.) H. Pfeiff., Sagittaria lancifolia L.] permiten separar esta asociación de los Llanos (Aristeguieta, 1968a; Castroviejo \& López, 1985; Susach Campalans, 1989) del SagittarioEleocharitetum interstinctae (Borhidi, 1996). Debido a la proximidad geográfica también incluimos en ésta última asociación los inventarios de Foucault (1981), de la isla de Guadalupe.

Las comunidades con Eleocharis interstincta (Vahl) Roem. \& Schult. y E. mutata están muy extendidas por toda la región del Caribe con distintas asociaciones de juncales que forman los "esteros". Aparte de Venezuela, tenemos datos de su presencia en las Antillas Menores (Loveless, 1960; Foucault, 1981), Cuba (Borhidi, 1996) y Bermudas (Prat, 1935). También se han descrito comunidades con Eleocharis interstincta en los Llanos de Moxos ( $\mathrm{N}$ de Bolivia)(Haase, 1989) aunque de composición florística diferente [Coutoubea ramosa Aubl., Eleocharis interstincta, Peritassa laevigata (Hoffmanns. ex Link) A.C. Sm.], por lo que probablemente haya que describir un nuevo orden para las sabanas situadas al $\mathrm{S}$ de la Cuenca Amazónica.

Thalietum geniculatae Castroviejo \& López 1985

[Tabla 8]

Asociación de grandes helófitos donde 
Tabla 7

1-5: Eleocharitetum mutatae Castroviejo \& López 1985

6-11: Eleocharitetum interstinctae Castroviejo \& López 1985

(Cladietea jamaicensis, Scirpo-Eleocharitetalia, Eleocharition interstincto-mutatae)

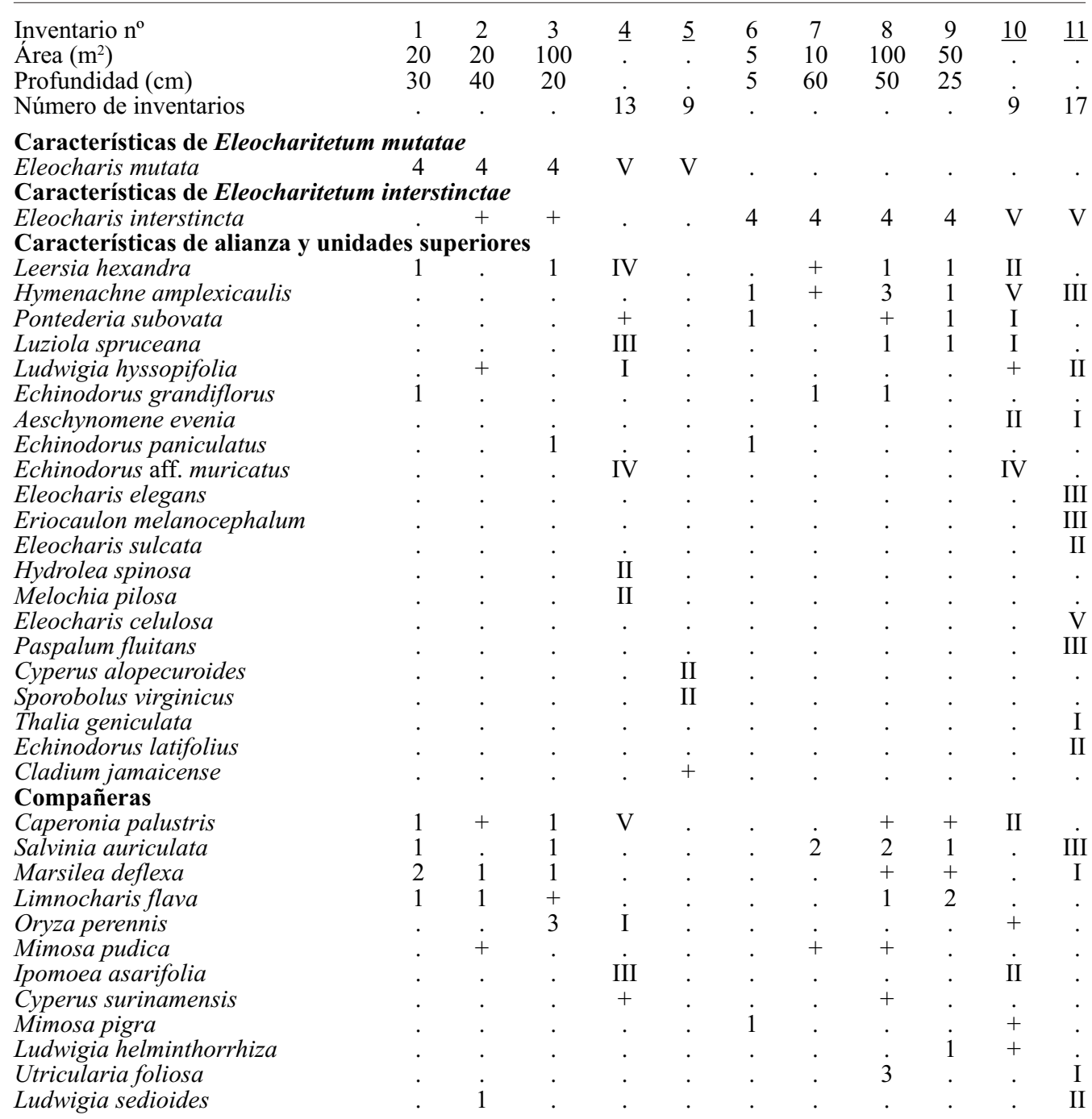

Otras plantas: Utricularia gibba + , Sida $\mathrm{sp} .+$, Utricularia hydrocarpa 1, Malachra radiata 1, Borreria $\mathrm{sp} .1$ en 1 ; Bacopa monnierioides + en 2; Maranta arundinacea +, Fimbristylis miliacea 1 en 3; Limnocharis sp. I, Sacciolepis myuros I, Spilanthes uliginosa +, Bacopa salzmanni + , Sagittaria planitiana + , Borreria $\mathrm{cf}$. aristeguietana II, Mimosa cf. pigra I, Borreria verticillata II, Aniseia martinicensis I en 4; Laguncularia racemosa + en 5; Solanum sp. +, Sapium glandulosum + en 6; Eichhornia crassipes 1 en 7; Nymphaea conardii +, Mimosa quadrivalvis subsp. leptocarpa + en 8; Eichhornia diversifolia + en 9; Cyperus sp. I, Hyptis pulegioides II en 10; Cyperus haspan II, Rhynchospora cyperoides ,+ Najas arguta I, Acisanthera limnobios I, Paratheria prostrata II, Buchnera palustris I, Nymphoides humboldtiana V, Eichhornia heterosperma I, Eriocaulon sp. I, Ludwigia octovalvis IV, Rhynchospora guianensis IV, Marsilea polycarpa II, Sagittaria guyanensis IV, Sinapea pratensis + en 11.

Localidades: 1- La Carmera; 2, 3- entrada del Hato El Frío; 4- Castroviejo \& López (1985, tab. 10, Venezuela); 5, Foucault (1981, tab. 5, Antillas Francesas); 6, 7, 8, 9- camino del Caño Mucuritas; 10- Castroviejo \& López (1985, tab. 9, Venezuela); 11- Susach Campalans (1989, tab. 24, Venezuela). 
domina Thalia geniculata L., que necesita suelos arcillosos y un nivel de inundación que puede llegar hasta $150 \mathrm{~cm}$ en la época de lluvias (Castroviejo \& López, 1985); en el período de sequía siempre presentan humedad edáfica.

$\mathrm{Su}$ estructura cerrada hace que no sea una asociación con elevada diversidad, aunque no deja de presentar elementos del Eleocharitetum interstinctae (Echinodorus paniculatus Micheli, Eleocharis interstincta) $\mathrm{u}$ otras plantas de medios acuáticos (Hymenachne amplexicaulis) que la acompañan (fig. 4).

Thalietum geniculatae, de los Llanos venezolanos y las Antillas, es vicariante del Rhabdadenio-Cyperetum gigantei chaqueño (Galán de Mera \& Navarro, 1992) que lleva Thalia multiflora Horkel. Igualmente se conocen comunidades de Thalia geniculata y Cyperus giganteus Vahl en el Pantanal de Mato Grosso (Zeilhofer \& Schess1, 1999).

Aunque en algunas zonas de América del Sur podemos encontrar comunidades de helófitos donde interviene Phragmites australis (Cav.) Steud. (PhragmitoMagnocaricetea Klika in Klika \& Novák 1941), parece ser, que al menos en el subreino Caribeo-Amazónico, se mantienen de forma constante algunas especies [Cladium jamaicense Crantz, Eleocharis interstincta, Gynerium sagittatum (Aubl.) P. Beauv., Hymenachne amplexicaulis, Montrichardia arborescens (L.) Schott] que nos inclinan a considerar la clase Cladietea jamaicensis Knapp ex Borhidi 1996.

\section{Luziolo spruceanae-Hymenachnetum \\ amplexicaulis Susach Campalans 1989, nom. inv.}

[Tabla 9; lectotypus: Susach Campalans (1989), tab. 25, inv. 184, elegido aquí]

Asociación caracterizada por Gramíneas, que desde las orillas arcillosas de los esteros forman una capa verde flotante en contacto con la vegetación de pleustohelófitos (fig. 4 y 6), a los que llega a desplazar.

\section{Comunidad de Xyris caroliniana y Eleocharis acutangula \\ [Tabla 9]}

Eleocharis acutangula (Roxb.) Schult. llega a formar turberas sobre suelos arenosos, donde además es capaz de desarrollarse Xyris caroliniana Walter y Osmunda cinnamomea L. Estos medios tan específicos, poco oxigenados, sólo permiten el asentamiento de algunas especies.

\section{Comunidad de Polygonum punctatum \\ [Tabla 9]}

Polygonum punctatum Elliott forma tapices sobre el agua, en encharcamientos eutrofizados como consecuencia de un fuerte estiaje. Este tipo de fitocenosis con especies afines del género Polygonum parecen tener una distribución bastante amplia en América del Sur; al menos, hemos detectado su presencia en la Amazonía peruana (Galán de Mera, 1995), el Chocó (Rangel, 2004) y en los Andes bolivianos (Antezana Valera, 2004) y colombianos (Wijninga et al., 1989).

Estas comunidades, compuestas de Gramíneas y de otros helófitos, se presentan formando extensiones por encima del agua, que sólo son características de los ambientes palustres tropicales y las incluimos en la alianza Hymanachnion amplexicaulis Galán de Mera 1995 y en el orden caribeoamazónico Oryzo grandiglumisHymenachnetalia amplexicaulis Galán de Mera \& Rosa in Galán de Mera, Rosa \& Cáceres 2002 que refleja la vegetación adaptada a las grandes fluctuaciones del nivel del agua (Beck, 1983; Haase, 1989; Susach Campalans, 1989; Galán de Mera, 1995; Fuentes \& Navarro, 2000). La 
Tabla 8

Thalietum geniculatae Castroviejo \& López 1985

(Cladietea jamaicensis, Typho-Cladietalia, Typhion domingensis)

\begin{tabular}{|c|c|c|c|c|}
\hline Inventario $\mathrm{n}^{\mathrm{o}}$ & 1 & 2 & 3 & $\underline{4}$ \\
\hline Área $\left(\mathrm{m}^{2}\right)$ & 10 & 50 & 100 & \\
\hline Número de inventarios & . & . & . & 3 \\
\hline \multicolumn{5}{|c|}{ Características de asociación y unidades superiores } \\
\hline Thalia geniculata & 5 & 4 & 4 & 3 \\
\hline Hymenachne amplexicaulis & 2 & 2 & & 3 \\
\hline Eleocharis interstincta & . & . & 1 & 1 \\
\hline Aeschynomene evenia & . & . & & 2 \\
\hline Ipomoea carnea subsp. fistulosa & . & . & 1 & . \\
\hline Leersia hexandra & . & . & 1 & . \\
\hline Ludwigia hyssopifolia & . & + & . & \\
\hline Echinodorus aff. muricatus & . & . & . & 1 \\
\hline Eleocharis elegans & . & . & & . \\
\hline Echinodorus paniculatus & . & . & 1 & . \\
\hline Eriocaulon melanocephalum & . & . & - & . \\
\hline Eleocharis sulcata & 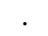 & . & . & \\
\hline Hydrolea spinosa & & . & . & 1 \\
\hline \multicolumn{5}{|l|}{ Compañeras } \\
\hline Salvinia auriculata & + & . & + & \\
\hline Caperonia palustris & 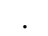 & . & . & 1 \\
\hline
\end{tabular}

Otras plantas: Ludwigia helminthorrhiza 1, Eichhornia azurea 1, Mikania micrantha + en 1; Malachra radiata +, Cyperus surinamensis,+ Borreria sp. + en 2; Mimosa pudica 1, Sida glomerata + en 3; Ipomoea asarifolia 1, Borreria verticillata 1, Eichhornia heterosperma 1 en 4; Nymphoides humboldtiana 2, Eriocaulon sp. 2, Ludwigia octovalvis 3, Utricularia foliosa 3, Rhynchospora guianensis 3, Marsilea polycarpa 2, Sagittaria guyanensis 3, Sipanea pratensis 2, Aeschynomene filosa 3 en $\underline{5}$.

Localidades: 1-3- entre la estación biológica y el aeropuerto; 4- Castroviejo \& López (1985, tab. 8); 5Susach Campalans (1989, tab. 23).

presencia casi constante de Hymenachne amplexicaulis es el denominador común, ya que se trata de un helófito que puede mostrarse prostrado o erguido cuando ha logrado invadir un espacio acuático cerrado (Rial, 2000).

Comunidades de Ipomoea carnea subsp. fistulosa

$$
\text { [Tabla 9] }
$$

Las comunidades de Ipomoea carnea subsp. fistulosa (Mart. ex Choisy) D.F. Austin están muy relacionadas con los asentamientos de la fauna silvestre de los Llanos. Generalmente responden a los reposaderos de las capibaras (Hydrochoeris hydrochoeris L.) en las orillas arenosas de los esteros, donde la nitrificación se hace evidente.

En la época de lluvias la comunidad se ve enriquecida con plantas flotantes, e incluso llega a reemplazar al Thalietum geniculatae cuando es alterado (Rial, 2000).

Este tipo de fitocenosis está muy extendida por el Neotrópico. Conocemos datos de Bolivia, donde Fuentes \& Navarro (2000) describen la asociación Mimoso chaetosphero-Ipomoeetum fistulosae y la llevan a la alianza provisional Ipomoeion fistulosae haciéndola extensiva a los humedales brasileño-paranenses; también las hemos observado en la Amazonía y en los bosques húmedos del $\mathrm{N}$ del Perú. 
Tabla 9

1-6: Luziolo spruceanae-Hymenachnetum amplexicaulis Susach 1989

7: Comunidad de Xyris caroliniana y Eleocharis acutangula

8: Comunidad de Polygonum punctatum

(Cladietea jamaicensis, Oryzo-Hymenachnetalia, Hymenachnion amplexicaulis)

9-10: Comunidad de Ipomoea carnea subsp. fistulosa

(Ipomoeion fistulosae)

$\begin{array}{lcccccccccc}\text { Inventario } \mathrm{n}^{\mathrm{o}} & 1 & 2 & 3 & 4 & 5 & \underline{6} & 7 & 8 & 9 & 10 \\ \text { Área }\left(\mathrm{m}^{2}\right) & 5 & 7 & 5 & 5 & 6 & . & 100 & 50 & 10 & 10 \\ \text { Profundidad }(\mathrm{cm}) & 80 & 90 & 90 & 70 & 70 & . & . & 80 & . & . \\ \text { Número de inventarios } & . & . & . & . & . & 6 & . & . & . & .\end{array}$

\section{Características de Luziolo-Hymenachnetum}

Luziola spruceana $2+$

Com. Xyris caroliniana $y$ Eleocharis acutangula

Xyris caroliniana

Eleocharis acutangula

Osmunda cinnamomea

Com. Polygonum punctatum

Polygonum punctatum

Com. Ipomoea carnea subsp. fistulosa

Ipomoea carnea subsp. fistulosa

Características de unidades superiores

Hymenachne amplexicauli

Leersia hexandra

Ludwigia hyssopifolia

Aeschynomene evenia

Thalia geniculata

Compañeras

Eichhornia crassipes

Salvinia auriculata

Panicum elephantipes

Caperonia palustris

Mimosa pudica

Ipomoea asarifolia

\begin{tabular}{|c|c|c|c|c|c|c|c|c|}
\hline 4 & 3 & 5 & 4 & 3 & $\mathrm{~V}$ & 1 & 1 & . \\
\hline . &. & . & . & . & $\cdot$ & . & 2 &. \\
\hline . & . & . & . & . & & $\cdot$ & r. & . \\
\hline . &. & . & . & . & IV & . & . & . \\
\hline . & $\cdot$ & . & . & . & $\cdot$ & . & . & . \\
\hline 1 & 2 & . & 1 & + & . & . & . & . \\
\hline+ & 1 & $\cdot$ & 2 & $\cdot$ & - & . & . & . \\
\hline+ & + & $\cdot$ & $\cdot$ & . & III & . & . & . \\
\hline 2 & 1 & . & . & . & . & $\cdot$ & . & . \\
\hline · & . & . & . & . & . & + & . & 1 \\
\hline . & $\cdot$ & $\cdot$ & $\cdot$ & . & . & . & . & + \\
\hline
\end{tabular}

Otras plantas: Utricularia foliosa 1 en 5; Paspalum fasciculatum IV, Bechnum serrulatum III, Axonopus anceps I, Sorghastrum parviflorum I, Scleria setacea III, Rhychospora podosperma II, Andropogon brevirostris II en 6, Andropogon bicornis + en 7; Ludwigia nervosa 2 en 8; Ambrosia cumanensis +, Evolvulus nummularius 1, Cynodon dactylon 2, Cyperus surinamensis 1, Sida glomerata 1 en 9; Mikania micrantha 1 en 10.

Localidades: 1, 2, 8, 9- camino del Caño Mucuritas; 3- entrada del Hato El Frío; 4,5- La Carmera; $\underline{6}$ Susach Campalans (1989, tab. 25); 7- Matagorda; 10- camino de Manirito.

5. Las comunidades de pleustohelófitos, pleustófitos e hidrófitos

(Clases Pistio-Eichhornietea, Lemnetea y Cabombo-Nymphaeetea)

Eichhornietum azureae Borhidi in Borhidi, Muñiz \& Del Risco 1983

[Tabla 10]
Comunidades dominadas por Eichhornia azurea (Sw.) Kunth, propias de aguas dulces oligótrofas o mesótrofas profundas (hasta $2 \mathrm{~m}$ ), con distribución neotropical (Castroviejo \& López, 1985; Borhidi, 1991; Galán de Mera \& Navarro, 1992). En los esteros es la vegetación acuática que se desarrolla a mayor profundidad.

La asociación Eichhornietum 
Tabla 10

1-7: Eichhornietum azureae Borhidi in Borhidi Muñiz \& Del Risco 1983

(Pistio-Eichhornietea, Pistio-Eichhornietalia, Eichhornion azureae)

8-17: Eichhornietum crassipedis Samek \& Moncada 1971

(Pistio-Eichhornietea, Pistio-Eichhornietalia, Pistio-Eichhornion)

18-19: Heteranthemetum limoso-reniformis Castroviejo \& López 1985

(Pistio-Eichhornietea, Pistio-Eichhornietalia, Heterantherion reniformis)

20-22: Comunidad de Lemna aequinoctialis

(Lemnetea minoris, Lemnetalia aequinoctialis, Azollo-Salvinion)

Inventario $\mathrm{n}^{\mathrm{o}}$

Área $\left(\mathrm{m}^{2}\right)$

Profundidad $(\mathrm{cm})$

Número de inventarios

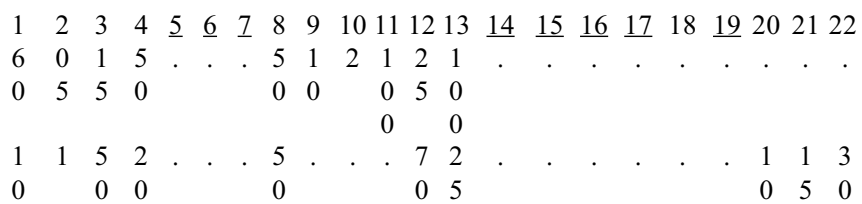

Características de Eichhornietum azureae y Eichhornion azureae

Eichhornia azurea $\begin{array}{lllllll}5 & 3 & 4 & 4 & \mathrm{~V} & \mathrm{~V}\end{array}$.

Características de Eichhornietum crassipedis y Pistio-Eichhornion

Eichhornia crassipes $\begin{array}{llllllllllllllllll}2 & 2 & 2 & \text {. } & \mathrm{I} & . & 3 & 4 & 2 & 5 & 5 & 2 & \mathrm{~V} & \mathrm{~V} & \mathrm{IV} & \mathrm{V} & + & \end{array}$.

Características de Heteranthemetum limoso-reniformis y Heteranthemion

Heteranthera limosa

Heteranthera reniformis

Características de Pistio-Eichhornietalia y Pistio-Eichhornietea

Pistia stratiotes

Panicum elephantipes

Eichhornia heterosperma

Neptunia prostrata

Ceratopteris pteridioides

Com. Lemna aequinoctialis, Lemnetea

Salvinia auriculata

Lemna aequinoctialis

Azolla caroliniana

Spirodela polyrrhiza

Lemna sp.

Azolla filiculoides

Lemna valdiviana

\section{Compañeras}

Hymenachne amplexicaulis

Leersia hexandra

Ludwigia hyssopifolia

Marsilea deflexa

Utricularia foliosa

Pontederia subovata

Mimosa pudica

Nymphoides humboldtiana

Ludwigia helminthorrhiza

Caperonia palustris

Ludwigia repens

Aeschynomene sensitiva

Nymphaea amazonum

Myriophyllum aquaticum

Utricularia gibba.

Hydrocotyle umbellata
2 . . . IV II . .

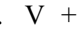

. IV +

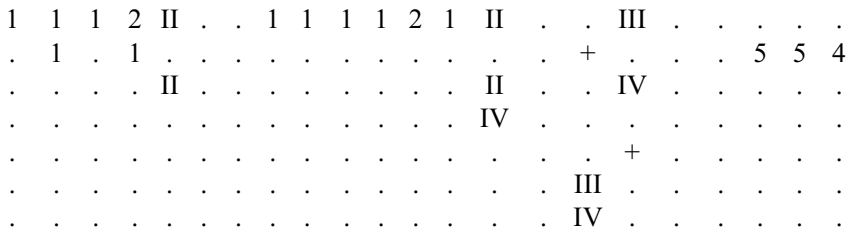

$\begin{array}{lllllllllllllllllllllll}2 & 1 & 2 & 2 & & . & \mathrm{V} & 4 & 1 & . & 1 & 2 & 1 & . & . & . & \mathrm{I} & . & . & 1 & 1 & 2\end{array}$

III

$1 \mathrm{~V}$

\begin{tabular}{cccccccccc}
. &. &. & II &. & 1 & 1 & 1 &. & 1 \\
\hline
\end{tabular}
II

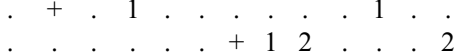

$+1 .+4+2$

. . . . I . . . I . . .

III

IV

II

III

IV 
Inventario $\mathrm{n}^{\mathrm{o}}$

Echinodorus paniculatus

Paspalum orbiculatum

Ludwigia inclinata.

Polygonum acuminatum

Ricciocarpus natans

Alternanthera crucis

Alternanthera aquatica

Sagittaria montevidensis

Pontederia cordata

Panicum repens

Spirodela intermedia

Salvinia rotundifolia

Cyperus giganteus

Paspalum repens

Heliotropium filiformis

Cabomba aquatica

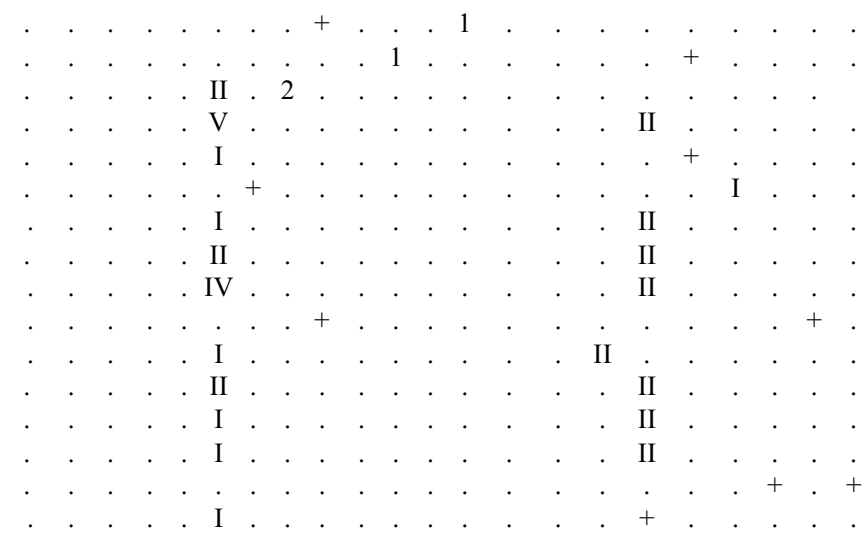

Otras plantas: Borreria sp. + en 1; Panicum aquaticum IV, Polygonum hispidum V, Polygonum hydropiperoides I en 5; Eleocharis sp. I, Aeschynomene sp. I, Scirpus californicus I, Loasa sp. I en $\underline{6}$; Eleocharis interstincta + , Oryza perennis I en 7; Kyllinga brevifolia +, Cuphea sp. + en 8; Echinodorus grandiflorus + , Solanum sp. + en 9; Hydrocleis grosourdyana 1 en 10; Luziola spruceana +,Ludwigia sedioides 1 en 11; Ludwigia nervosa 3 en 12; Cabomba piauhyensis 3; Maranta arundinacea + en 13; Paspalidium geminatum IV, Vigna sp. II en 14; Hydrocotyle ranunculoides IV, Scirpus americanus I, Ludwigia adscendens I, Enhydra maritima II, Typha domingensis + , Paspalum vaginatum + , Apium graveolens +, Nasturtium officinale +, Cyperaceae sp. + en $\underline{16}$; Hydrocleis nymphoides I, Marsilea polycarpa + , Aeschynomene virginica II, Sesbania virgata + , Pontederia rotundifolia I en 17; Hyptis brevipes + , Cynodon dactylon 1 en 18; Limnocharis sp. V, Aeschynomene evenia I, Fimbristylis miliacea II, Spilanthes uliginosa II, Sagittaria planitiana II, Fimbristylis aff. dichotoma I, Borreria cf. aristeguietana II, Cyperus surinamensis II en 19; Cassia tora + en 20; Ludwigia peploides I en 21.

Localidades: 1-4, 11, 12- de la estación biológica al Caño Guaritico; $\underline{5}$ - Borhidi (1991, tab. 47, Cuba); $\underline{6}$, 17- Galán de Mera \& Navarro (1992, tab. 4, Paraguay); 7, 19- Castroviejo \& López (1985, tab. 6, Venezuela); 8- entre la estación biológica y el aeropuerto, 9-10- aeropuerto; 13- entrada del Hato El Frío; 14, 15- Foucault (1981, tab. 4, Antillas Francesas); 16- Müller \& Gutte (1985, tab. 10, Perú); 18, 20-22- estación biológica.

heterospermo-azureae Castroviejo \& López 1985 , por su composición florística, es un sinónimo de ésta.

Eichhornietum crassipedis Samek \& Moncada 1971

[Tabla 10]

El aumento de la eutrofización de las aguas es lo que permite distinguir esta asociación de la anterior. Por esta razón, en el territorio estudiado, Eichhornietum crassipedis forma una banda hacia la orilla de las lagunas y esteros por fuera del Eichhornietum azureae, donde existe una mayor exposición a la nitrificación animal.

Tanto E. crassipes como E. azurea tienen un gran valor ecológico ya que sirven de alimento a los grandes roedores acuáticos $\mathrm{y}$, sus raíces fasciculadas, sirven de plataforma para el desove de algunos peces (Granja e Barros, 1980).

En ocasiones, cuando continua la eutrofización de las aguas, a veces por estiaje, E. crassipes comienza a ser sustituida por Pistia stratiotes L., que llega a formar una fenofase monoespecífica.

Heterantheretum limoso-reniformis Castroviejo \& López 1985

[Tabla 10]

De las asociaciones de la clase, es la que soporta una mayor desecación y nitrificación, 
incluso la podemos encontrar en caminos que han soportado nitrificación solamente durante la época de lluvias.

Se muestra en contacto con Eichhornietum crassipedis o con los pastizales de Spilantho-Paspaletum por lo que Ludwigia hyssopifolia y Paspalum orbiculatum también pueden formar parte de su composición.

Esta situación fugaz de Heteranthera limosa (Sw.) Willd. y H. reniformis Ruiz \& Pav. ya ha sido puesta de manifiesto en otras ocasiones (Galán de Mera, 1994) por lo que la separamos de otras comunidades de PistioEichhornietea mediante la alianza Heterantherion reniformis Galán de Mera, González, Morales, Oltra \& Vicente Orellana all. nova (holotypus: Heterantheretum limoso-reniformis Castroviejo \& López 1985).

\section{Comunidad de Lemna aequinoctialis [Tabla 10]}

Las lentejas de agua forman comunidades flotantes en las lagunas poco profundas (5-30 cm) y fuertemente eutrofizadas del área estudiada.

En esta ocasión Lemna aequinoctialis Welw. forma comunidades monoespecíficas, sin que intervenga otro pleustófito, aunque por la presencia de Salvinia auriculata y Azolla caroliniana Willd. en el territorio, no dudamos en incluirlas en la alianza neotropical Azollo carolinianae-Salvinion auriculatae Borhidi \& Muñiz in Borhidi 1996 (Landolt, 1999).

\section{Ludwigio sedioidis-Eichhornietum} diversifoliae Castroviejo \& López 1985 [Tabla 11]

Es la asociación central con plantas de hojas flotantes de los Llanos del Orinoco [Eichhornia diversifolia (Vahl) Urb., Hydrocleis grosourdyana Pedersen, Ludwigia sedioides (Bonpl.) Hara, Marsilea deflexa A. Braun, Nymphaea conardii Wiersema, Nymphoides humboldtiana (Kunth) Kuntze, Sagittaria guyanensis Kunth].

Es capaz de soportar grandes diferencias de inundación, desde 20 a $100 \mathrm{~cm}$, por lo que a lo largo del año va a presentar aspectos bien diferenciados. De acuerdo con los estudios de Colonnello (1995), en un primer estadio, las aguas ácidas con ácidos orgánicos, que son arrastradas desde los bosques adyacentes -estadio de aguas negras- permiten la instalación de plantas con hojas sumergidas (Utricularia foliosa L., U. gibba L., U. hydrocarpa Vahl). El aporte de lodos estadio de aguas blancas- al transcurrir la época de lluvias, permite la proliferación de Marsilea deflexa, que la podemos encontrar en pequeños charcos, para posteriormente dar paso al desarrollo de los ninfeidos (fig. $5)$, que ofrecen un gran colorido a las comunidades acuáticas de los Llanos entre octubre y diciembre. Este proceso permite explicar la riqueza florística de esta asociación y su heterogeneidad en distintas localidades.

Por tanto, consideramos a la asociación Sagittario-Marsileetum deflexae Castroviejo \& López 1985, y a las comunidades de Utricularia como fases sucesionales del Ludwigio-Eichhornietum diversifoliae. Se trata de una asociación vicariante de las comunidades con Nymphoides, Nymphaea, Limnocharis flava (L.) Buchenau y Nelumbo lutea Willd. del Caribe (Borhidi, 1996) y del Hydrocleido-Nymphoidetum humboldtianae (=Elodeo-Nymphaeetum amazoni) del Chaco (Galán de Mera \& Navarro, 1992). La alianza Sagittarion guyanensis Castroviejo \& López 1985 , por sus características y areal, se hace sinónima de Nelumbo-Nymphaeion amplae Samek \& Moncada 1971, descrita en Cuba.

6. Las comunidades nitrófilas

(Clases Sido-Stachytarphetaetea indicae y Polygono-Poetea) 
Tabla 11

Ludwigio sedioidis-Eichhornietum diversifoliae Castroviejo \& López 1985 (Cabombo-Nymphaeetea, Nymphaeetalia amplae, Nelumbo-Nymphaeion)

\begin{tabular}{|c|c|c|c|c|c|c|c|c|c|c|c|c|c|c|}
\hline Inventario $\mathrm{n}^{\mathrm{o}}$ & 1 & 2 & 3 & 4 & 5 & 6 & 7 & 8 & 9 & 10 & 11 & 12 & $\underline{13}$ & 14 \\
\hline Área $\left(\mathrm{m}^{2}\right)$ & 25 & 5 & 2 & 200 & 10 & 4 & 2 & 50 & 50 & 100 & 25 & 15 & & \\
\hline Profundidad $(\mathrm{cm})$ & 25 & 80 & 70 & 60 & 60 & 60 & ${ }^{\circ}$ & 50 & 90 & 1 & 60 & 50 & & \\
\hline Número de inventarios & & & 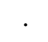 & & & . & . & & . & . & & . & 19 & \\
\hline \multicolumn{15}{|c|}{ Características de asociación y unidades superiores } \\
\hline Hydrocleis grosourdyana & & & 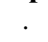 & 1 & + & 1 & 1 & 3 & 4 & 4 & & . & I & \\
\hline Ludwigia sedioides & & & + & 1 & 1 & 1 & & & 2 & 2 & & . & I & \\
\hline Marsilea deflexa & 3 & 1 & 1 & . & . & . & . & 1 & 1 & 1 & & . & V & \\
\hline Utricularia foliosa & 4 & 3 & 4 & & & & & 1 & . & & 1 & + & . & \\
\hline Nymphoides humboldtiana & & & . & 1 & 2 & 2 & 3 & . & . & 3 & & . & + & \\
\hline Sagittaria guyanensis & 1 & & & $\cdot$ & . & - & . & . & - & . & 2 & & V & \\
\hline Nymphaea conardii & & & 2 & & r & . & & . & 1 & . & 2 & 3 & . & \\
\hline Eichhornia diversifolia & 2 & 4 & . & . & . & . & 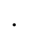 & . & 1 & . & . & . & . & \\
\hline Limnocharis flava & . & & . & & . & . & & & 1 & 1 & & . & & \\
\hline Cabomba piauhyensis & . & & . & & . & . & & r & . & 2 & & . & . & \\
\hline Utricularia gibba & . & & . & . & . & . & . & . & . & 1 & & . & . & \\
\hline
\end{tabular}

\section{Compañeras}

Hymenachne amplexicaulis

Salvinia auriculata

Ludwigia helminthorrhiza

Leersia hexandra

Paspalum orbiculatum

Echinodorus grandiflorus

Luziola spruceana

Ludwigia hyssopifolia

Eleocharis interstincta

Echinodorus paniculatus

Caperonia palustris

Solanum sp.

Eleocharis mutata

Sida sp.

Eichhornia crassipes

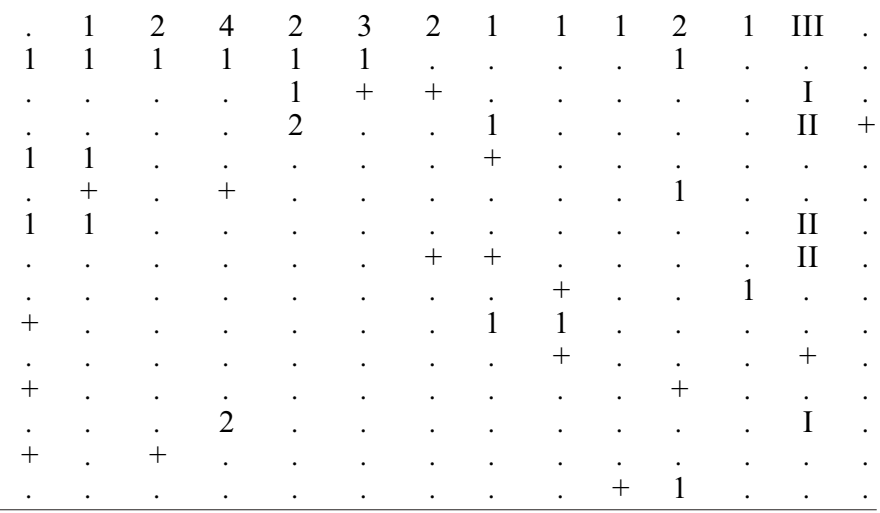

Otras plantas: Mimosa pudica +, Borreria sp. 1 en 1; Eichhornia azurea 2 en 2; Utricularia hydrocarpa 1, Nitella sp. 2 en 4; Eichhornia heterosperma 1 en 5; Ipomoea carnea subsp. fistulosa 1 en 8; Malachra radiata + en 9; Oryza perennis 1, Panicum repens + en 10; Sida procumbens + en 11; Justicia sp. +, Cassia aculeata 1, Pontederia subovata 1 en 12; Ipomoea asarifolia 1, Hydrolea spinosa 1, Bacopa salzmanni 1, Luziola pittieri + , Sida linifolia + , Heteranthera limosa 1, Alternanthera crucis II, Limnocharis sp. III, Aeschynomene evenia + en 13.

Localidades: 1-7- entre la estación biológica y el Caño Guaritico; 8, 12 - Matagorda; 9- entrada del Hato El Frío; 10- Mata Silva; 11- estación biológica; 13, 14- Castroviejo \& López (1985, tab. 13); 14 Castroviejo \& López (1985, tab. 7).

Sido glomeratae-Cassietum

torae Castroviejo \& López 1985 [Tabla 12]

Comunidad terofítica nitrófila muy extendida por todo el territorio en las zonas con excesiva acción de la ganadería, bordes de caminos y proximidades de casas, donde podemos observar los suelos removidos con una mayor abundancia de Cassia tora L., Cleome spinosa Jacq., Sida glomerata y Wissadula periplocifolia $\mathrm{C}$. Presl, aunque en zonas donde hay acumulación de lodos y se forman taludes, domina Hyptis suaveolens 


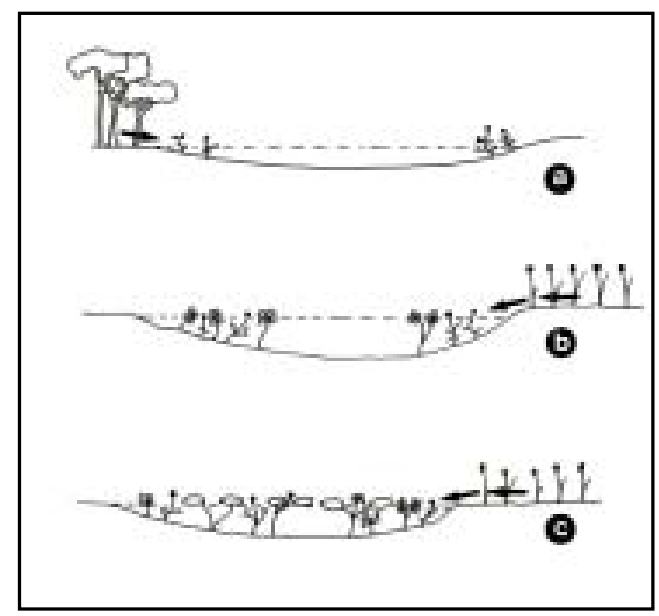

Figura 5. Esquema con la sucesión de fases de la asociación Ludwigio sedioidis-Eichhornietum diversifoliae: a) Arrastre de agua desde los bosques (fase de Utricularia), b) entrada de lodos por arrastre (fase de Marsilea deflexa), c) aumento del nivel de inundación (fase óptima de la asociación). Succession scheme of the association Ludwigio sedioidis-Eichhornietum diversifoliae: a) Water dragging from forest (Utricularia stage), b) muddy entrance by dragging (Marsilea deflexa stage), c) increase of flooding level (optimum stage of the association).

(L.) Poit. (hyptetosum suaveolentis Castroviejo \& López 1985). Tiene una gran importancia en el territorio estudiado debido a la explotación extensiva con ganado vacuno, que en época de lluvias queda restringida a ciertas zonas más elevadas, llegando a desplazar a los pastizales de Elionurion tripsacoidis (Ramia, 1972).

Desde el punto de vista sintaxonómico, podemos incluir esta asociación en la alianza provisional caribeño-mesoamericana Wissadulo periplocifoliae-Cassion torae Galán de Mera, González, Morales, Oltra \& Vicente Orellana all. nova prov., en el orden Eleusinetalia indicae Knapp 1957, y en la clase nitrófila caribeo-amazónica de sabanas secundarias Sido-Stachytarphetaetea indicae Hoff in Hoff, Brisse \& Grandjouan 1983 (Hoff \& Brisse, 1983).

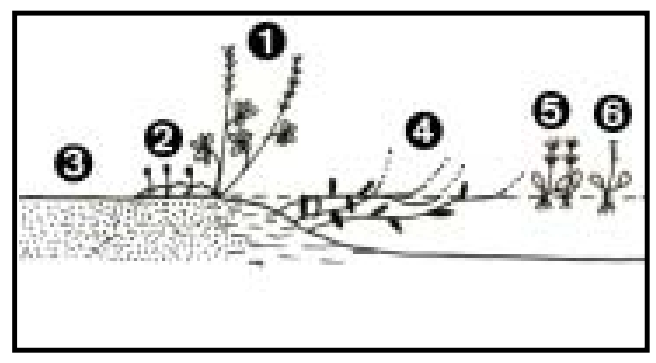

Figura 6. Esquema de la vegetación nitrófila en el territorio estudiado. Scheme of the nitrophilous vegetation in the studied territory. 1-Sido glomeratae-Cassietum torae, 2- Comunidad de Evolvulus nummularius y Alternanthera crucis, 3- Camino, 4- Luziolo-Hymenachnetum amplexicaulis, 5- Eichhornietum crassipedis, 6Eichhornietum azureae.

Comunidad de Evolvulus nummularius y Alternanthera crucis

[Tabla 12]

Comunidad que se muestra en contacto con la anterior en los bordes de caminos (fig. 6), donde tanto Evolvulus nummularius (L.) L. como Alternanthera crucis Bold. resisten el pisoteo de la ganadería en los caminos que utilizan los llaneros para transportar cebúes. Ambas plantas a veces van acompañadas de Cynodon dactylon (L.) Pers., lo que resalta la ecología de esta comunidad que, de momento, incluimos en la clase cosmopolita Polygono-Poetea annuae Rivas-Martínez 1975.

Geophiletum repentis Galán de Mera, González, Morales, Oltra \& Vicente Orellana ass. nova

[Tabla 12, holotypus ass. inv. 11]

Asociación casi monoespecífica dominada por Geophila repens (L.) I.M. Johnst., pequeña Rubiácea nemoral de los bosques húmedos adaptada a los aportes nitrogenados de la hojarasca y pequeños 
Tabla 12

1-7: Sido-Cassietum torae Castroviejo \& López 1985

(Sido-Stachytarphetaetea, Eleusinetalia indicae, Wissadulo-Cassion)

8-10: Comunidad de Evolvulus nummularius y Alternanthera crucis (Polygono-Poetea annuae)

11-12: Geophiletum repentis ass. nova

$\begin{array}{lcccccccccccc}\text { Inventario } \mathrm{n}^{\mathrm{o}} & 1 & 2 & 3 & 4 & 5 & 6 & \underline{7} & 8 & 9 & 10 & 11 & 12 \\ \text { Área }\left(\mathrm{m}^{2}\right) & 100 & 100 & 100 & 100 & 100 & \dot{7} & \dot{8} & 2 & 6 & 12 & 10 & 5 \\ \text { Número de inventarios } & & & & & & 7 & \end{array}$

Características de Sido-Cassietum torae y unidades superiores

Sida glomerata

Cassia tora

Wissadula periplocifolia

Heliotropium indicum

Cleome spinosa

Ambrosia cumanensis

Sida acuta

Sida procumbens

Cuphea elliptica

Pavonia sessiliflora

Murdannia nodiflora

Sida glomerata $x$ acuta

Synedrella nodiflora

Eleusine indica

Amaranthus spinosus

Diferencial de hyptetosum suaveolentis

Hyptis suaveolens

Com. Evolvulus nummularius y Alternanthera crucis

Evolvulus nummularius

Alternanthera crucis

Cynodon dactylon

Ipomoea asarifolia

Características de Geophiletum repentis

Geophila repens

Pharus latifolius

Compañeras

Mimosa pigra

Mimosa pudica

Borreria aristeguietana

Solanum hirtum

Calopogonium mucunoides

Axonopus compressus

Desmodium scorpiurus

Borreria verticillata

Eichhornia crassipes

Piper tuberculatum

Psychotria anceps

Borreria latifolia

Euphorbia cf. dioica

$\begin{array}{ccccccc}1 & 1 & + & + & 1 & \text { V } & \text { V } \\ 4 & 4 & 5 & 5 & 4 & \text { V } & \text { IV } \\ 1 & + & 1 & 1 & 1 & \text { III } & . \\ 2 & 1 & 2 & 2 & 1 & . & . \\ 2 & 2 & 1 & + & 1 & . & . \\ 1 & 2 & 4 & + & . & . & . \\ . & . & . & . & . & \text { III } & \text { V } \\ . & . & + & + & . & . & \text { I } \\ . & . & . & . & . & \text { III } & \text { III } \\ . & . & . & . & . & \text { I } & \text { IV } \\ . & . & . & . & . & \text { III } & \text { II } \\ . & . & . & . & . & \text { II } & \text { II } \\ . & . & . & . & . & \text { III } & \text { I } \\ . & . & . & . & . & \text { I } & \text { I } \\ . & . & . & . & . & . & . \\ . & . & . & . & + & . & .\end{array}$

Otras plantas: Paspalum orbiculatum I, Hyptis pulegioides I, Aeschynomene brasiliana I, Lindernia crustacea I, Cyperus luzulae 1 en 6. Euphorbia hyssopifolia II, Melochia parvifolia II, Cyperus sphacelatus II, Momordica sp. II, Cyperus sp. II, Marsypianthes sp. I, Kyllinga odorata +, Elionurus tripsacoides +, Cenchrus pilosus 1, Dichromena ciliata I, Urera sinuata I, Mimosa orthocarpa 1, Ipomoea sp. I, Hyptis mutabilis I, Diodia teres subsp. prostrata I en 7; Brachiaria fasciculata I, Hyptis brevipes + en 8; Manguifera indica + en 9; Ludwigia inclinata + en 10.

Localidades: 1-5- camino de Manirito; 6- Castroviejo \& López (1985, tab. 15); 7- Castroviejo \& López (1985, tab. 15); 8-10- de la estación biológica al Caño Macanillal; 11, 12- La Carmera. 
animales que favorecen el ahuecado de los suelos.

Es una asociación de amplia distribución neotropical (Steyermark, 1974), que también hemos podido observar en la Amazonía peruana aunque, por el momento, no la podemos incluir en ninguna unidad sintaxonómica superior.

\section{CONCLUSIONES}

Como conclusión damos a conocer el esquema sintaxonómico comentado del territorio estudiado, según las formaciones vegetales reconocidas:

1. Bosques y arbustedas

CEIBETEA OCCIDENTALIS Knapp ex Borhidi 1996

+ Bactrido guineensis-Cecropietalia peltatae Galán de Mera, González, Morales, Oltra \& Vicente Orellana ordo novo

* Desmonco orthacanthi-Platymiscion pinnati Galán de Mera, González, Morales, Oltra \& Vicente Orellana all. nova

1. Bromelio chrysanthae-Platymiscietum pinnati Galán de Mera, González, Morales, Oltra \& Vicente Orellana ass. nova [Bosques semidecíduos de los Llanos Occidentales del Orinoco]

platymiscietosum pinnati

copernicietosum tectorum Galán de Mera, González, Morales, Oltra \& Vicente Orellana subass. nova [Bosques con palmeras llaneras]

duguetietosum riberensis Galán de Mera, González, Morales, Oltra \& Vicente Orellana subass. nova [Bosques con crecidas estacionales]

* Nectandro globosae-Viticion orinocensis Galán de Mera, González, Morales, Oltra \& Vicente Orellana all. nova
2. Randio venezuelensis-Annonetum jahnii Galán de Mera, González, Morales, Oltra \& Vicente Orellana ass. nova [Arbustedas sobre suelos limo-arenosos] 3. Helictero guazumifoliae-Bauhinietum benthamianae Galán de Mera, González, Morales, Oltra \& Vicente Orellana ass. nova [Arbustedas sobre arcillas amarillas]

\section{Arbustedas ribereñas}

COCCOLOBIETEA OBTUSIFOLIAE Galán de Mera, González, Morales, Oltra \& Vicente Orellana cl. nova

+ Coccolobietalia obtusifoliae Galán de Mera, González, Morales, Oltra \& Vicente Orellana ord. novo

* Coccolobion obtusifoliae Galán de Mera, González, Morales, Oltra \& Vicente Orellana all. nova

4. Coccolobetum obtusifoliae Castroviejo \& López 1985 [Arbustedas de ríos con gran caudal]

3. Pastizales

LEPTOCORYPHIO-TRACHYPOGONETEA Van Donselaar 1965

+ Trachypogonetalia plumosi Van Donselaar 1965

* Elionurion tripsacoidis Castroviejo \& López 1985

5. Cassio rotundifoliae-Elionuretum tripsacoidis Castroviejo \& López 1985 [Pastizales no inundables]

6. Panico juncei-Imperatetum contractae Castroviejo \& López 1985

[Pastizales con ligera inundación]

XYRIDETEA SAVANENSIS Galán de Mera 1995

+ Eleocharitetalia minimae Galán de Mera, González, Morales, Oltra \& Vicente Orellana ord. novo

* Oryzion perennis Galán de Mera, 
González, Morales, Oltra \& Vicente Orellana all. nova

7. Spilantho uliginosi-Paspaletum orbiculati Castroviejo \& López 1985 [Pastizales inundables]

\section{Comunidades helofíticas}

CLADIETEA JAMAICENSIS Knapp ex Borhidi 1996

+ Scirpo-Eleocharitetalia interstinctae Borhidi \& Muñiz in Borhidi 1996

* Eleocharition interstincto-mutatae Castroviejo \& López 1985

8. Eleocharitetum mutatae Castroviejo \& López 1985 [Juncal de aguas poco profundas]

9. Eleocharitetum interstinctae Castroviejo \& López 1985 [Juncal de aguas profundas] + Typho-Cladietalia jamaicensis Borhidi \& Del Risco in Borhidi 1996

* Typhion domingensis Del Risco in Borhidi 1996

10. Thalietum geniculatae Castroviejo \& López 1985 [Asociación de grandes helófitos sobre suelos arcillosos]

+ Oryzo grandiglumis-Hymenachnetalia amplexicaulis Galán de Mera \& Rosa in Galán de Mera, Rosa \& Cáceres 2002

* Hymenachnion amplexicaulis Galán de Mera 1995

11. Luziolo spruceanae-Hymenachnetum amplexicaulis Susach Campalans 1989 [Graminales flotantes]

12. Comunidad de Xyris caroliniana y Eleocharis acutangula [Turberas sobre suelos arenosos]

13. Comunidad de Polygonum punctatum [Tapices de Polygonáceas sobre aguas eútrofas].

* Ipomoeion fistulosae Fuentes \& Navarro 2000, prov.

14. Comunidades de Ipomoea carnea subsp. fistulosa [Vegetación hidronitrófila de reposaderos]
5. Comunidades de pleustohelófitos, helófitos e hidrófitos

PISTIO STRATIOTIDIS-EICHHORNIETEA CRASSIPEDIS O. Bolòs, Cervi \& Hatschbach 1991

+ Pistio stratiotidis-Eichhornietalia crassipedis O. Bolòs, Cervi \& Hatschbach 1991

* Pistio stratiotidis-Eichhornion crassipedis

O. Bolòs, Cervi \& Hatschbach 1991

15. Eichhornietum crassipedis Samek \& Moncada 1971 [Pleustohelófitos de aguas eútrofas]

* Eichhornion azureae Borhidi \& Muñiz ex Galán de Mera \& Navarro 1992

16. Eichhornietum azureae Borhidi in Borhidi, Muñiz \& Del Risco 1983 [Pleustohelófitos de aguas dulces oligótrofas o mesótrofas]

* Heterantherion reniformis Galán de Mera, González, Morales, Oltra \& Vicente Orellana all. nova

17. Heterantheretum limoso-reniformis Castroviejo \& López 1985 [Pleustohelófitos fugaces]

CABOMBO-NYMPHAEETEA Borhidi \& Del Risco in Borhidi, Muñiz \& Del Risco (1979) 1983

+ Nymphaeetalia amplae Knapp ex Borhidi 1996

* Nelumbo-Nymphaeion amplae Samek \& Moncada 1971 [Sin.: Sagittarion guyanensis Castroviejo \& López 1985]

18. Ludwigio sedioidis-Eichhornietum diversifoliae Castroviejo \& López 1985 [incl. Sagittario-Marsileetum deflexae Castroviejo \& López 1985] [Comunidades de ninfeidos de los Llanos del Orinoco]

\section{Comunidades nitrófilas}

SIDO-STACHYTARPHETAETEA INDICAE Hoff in Hoff, Brisse \& Grandjouan 1983 + Eleusinetalia indicae Knapp 1957 
* Wissadulo periplocifoliae-Cassion torae Galán de Mera, González, Morales, Oltra \& Vicente Orellana all. nov. prov.

19. Sido glomeratae-Cassietum torae Castroviejo \& López 1985 [Vegetación nitrófila antropozoógena]

cassietosum torae

hyptetosum suaveolentis Castroviejo \& López 1985 [Subasociación de suelos con abundancia de lodos]

\section{POLYGONO ARENASTRI-POETEA ANNUAE Rivas-Martínez 1975}

20. Comunidad de Evolvulus nummularius y Alternanthera crucis [Comunidades de suelos pisoteados]

21. Geophiletum repentis Galán de Mera, González, Morales, Oltra \& Vicente Orellana ass. nova [Asociación nemoral de bosques húmedos]

AGRADECIMIENTOS. Agradecemos al Dr. Javier Castroviejo Bolíbar su constante estímulo en la realización de trabajos sobre flora y vegetación de Venezuela. Gracias también al personal de la estación biológica El Frío y a la Fundación Amigos de Doñana, en el marco del máster en Gestión y Conservación de la Biodiversidad en Los Trópicos (Fundación Carolina-Fundación Amigos de DoñanaUniversidad San Pablo-CEU), cuya ayuda logística fue fundamental.

\section{BIBLIOGRAFÍA}

ANDERSON, A.B., G.T. PRANCE \& B.W.P. ALBURQUERQUE -1975- Estudos sobre a vegetação das Campinas Amazonicas- III. A vegetação lenhosa da Campina da Reserva Biológica INPA-SUFRAMA (ManausCaracaraí, Km 62). Acta Amazonica 5: 225246.

ANTEZANA VALERA, C.F. -2004- Estudio de la flora y la vegetación de los valles secos interandinos del departamento de
Cochabamba (Bolivia). Universidad Complutense de Madrid.

ARISTEGUIETA, L. -1968a- Consideraciones sobre la flora de los morichales llaneros al norte del Orinoco. Acta Bot. Ven. 3: 19-38.

ARISTEGUIETA, L. -1968b- El bosque caducifolio seco de los Llanos Altos Centrales. Bol. Soc. Venez. Ci. Nat. 27: 395438.

ARISTEGUIETA, L. -1973- Familias y Géneros de los Árboles de Venezuela. Caracas.

BECK, S.G. -1983- Vegetationsökologische Grundlagen der Viehwirtschaft in den Überschwemmungs-Savannen des Río Yacuma (Departamento Beni, Bolivien). Diss. Bot. 80: 1-186.

BLYDENSTEIN, J. -1961- La vegetación de la Estación Biológica de Los Llanos. Bol. Soc. Ven. Ci. Nat. 22: 208-212.

BLYDENSTEIN, J. -1962- La sabana de Trachypogon del Alto Llano (Estudio ecológico de la región alrededor de Calabozo, Estado Guárico). Bol. Soc. Ven. Ci. Nat. 23: 139-206.

BLYDENSTEIN, J. -1963- La vegetación en el estero del río Guariquito. Bol. Soc. Ven. Ci. Nat. 23: 229-232.

BOLÒS, O., A.C. CERVI y G. HATSCHBACH -1991- Estudios sobre la vegetación del estado de Paraná (Brasil meridional). Collect. Bot. (Barcelona) 20: 79-182.

BORHIDI, A. -1991- Phytogeography and Vegetation Ecology of Cuba. Budapest.

BORHIDI, A. -1996- Phytogeography and Vegetation Ecology of Cuba. Budapest.

BORHIDI, A., O. MUÑIZ y E. DEL RISCO 1979- Clasificación fitocenológica de la vegetación de Cuba. Acta Bot. Acad. Sci. Hung. 25: 263-301.

BORHIDI, A., O. MUÑIZ \& E. DEL RISCO 1983- Plant communities of Cuba, I. Fresh and salt water, swamp and coastal vegetation. Acta Bot. Hung. 29: 337-376.

BRAUN-BLANQUET, J. -1964Pflanzensoziologie, Grundzüge der Vegetationskunde. Wien.

CASTILLO S.A. -1977- Estudio de una sección del bosque de galería del Río Orituco al sur de los Llanos de Calabozo. Universidad Central de Venezuela. Caracas. 
CASTROVIEJO, S. y G. LÓPEZ -1985- Estudio y descripción de las comunidades vegetales del "Hato El Frío". Los Llanos de Venezuela. Mem. Soc. Ci. Nat. La Salle 45: 79-151.

CLARK, H., R. LIESNER, P.E. BERRY, A. FERNÁNDEZ, G. AYMARD y P. MAQUIRINO -2000- Catálogo anotado de la flora del área de San Carlos de Río Negro, Venezuela. Scientia Guianae 11: 101-316.

COLONNELLO, G. -1995- La vegetación acuática del delta del río Orinoco (Venezuela). Composición florística y aspectos ecológicos (I). Mem. Soc. Ci. Nat. La Salle 55: 3-34.

COLONNELLO, G., S. CASTROVIEJO y G. LÓPEZ -1986-Comunidades vegetales asociadas al Río Orinoco en el sur de Monagas y Anzoátegui (Venezuela). Mem. Soc. Ci. Nat. La Salle 46: 127-165.

COMERMA G., J.A. y M. O. LUQUE -1971- Los principales suelos y paisajes del Estado de Apure. Agron. Trop. 21: 365-377.

CUELLO, N., G. AYMARD y B. STERGIOS 1989- Observaciones sobre la vegetación de un sector de la cuenca media del río Portuguesa, Estado Portuguesa, Venezuela. Biollania 6: 163-192.

DUIVENVOORDEN, J.F. \& A.M. CLEEF -1994Amazonian savanna vegetation on the sandstone plateau near Araracuara, Colombia. Phytocoenologia 24: 197-232.

ENCARNACIÓN, F. -1985- Introducción a la flora y vegetación de la Amazonía peruana: estado actual de los estudios, medio natural y ensayo de una clave de determinación de las formaciones vegetales en la llanura amazónica. Candollea 40: 237-252.

FOUCAULT, B. -1981- Nouvelles observations phytosociologiques sur la vegetation aquatique à la Guadeloupe (Antilles Francaises): de la vegetation phanerogamique marine aux bombements à sphaignes culminaux. Col. phytosoc. 10: 255-277.

FUENTES, A. y G. NAVARRO -2000- Estudio fitosociológico de la vegetación de una zona de contacto Chaco-Cerrado en Santa Cruz (Bolivia). Lazaroa 21: 73-109.

GALÁN DE MERA, A. -1994- Sinopsis de las Pontederiáceas del Perú. Arnaldoa 2: 47-56.

GALÁN DE MERA, A. -1995- Ensayo sintaxonómico sobre las comunidades vegetales acuáticas del Perú. Arnaldoa 3 : 5158.

GALÁN DE MERA, A. -2001a- Una aproximación fitosociológica sobre los varillales húmedos de la Amazonía peruana. Stud. bot. 20: 125-133.

GALÁN DE MERA，A. -2001b- Nota sintaxonómica sobre la vegetación del Chaco Boreal. Arnaldoa 8: 81-84.

GALÁN DE MERA, A. y G. NAVARRO -1992Comunidades vegetales acuáticas del Paraguay occidental. Caldasia 17: 35-46.

GALÁN DE MERA, A., M.V. ROSA y C. CÁCERES -2002- Una aproximación sintaxonómica sobre la vegetación del Perú. Clases, órdenes y alianzas. Acta. Bot. Malacitana 27: 75-103.

GENTRY, A.H. -1982-Bignoniaceae. Flora de Venezuela. Caracas.

GRANJA E BARROS, M.A. -1980- Estudo de macrófitas de agua doce coletadas en Brasilia (D.F.). Anales Soc. Brot. 46: 27-40.

HAASE, R. -1989- Plant communities of a savanna in northern Bolivia I. Seasonally flooded grassland and gallery forest. Phytocoenologia 18: 55-81.

HAASE, R. -1990- Plant communities of a savanna in northern Bolivia II. Palm swamps, dry grassland, and shrubland. Phytocoenologia 18: 343-370.

HOFF, M. \& H. BRISSE -1983- Proposition d'un schema synthetique des vegetations secundaires intertropicales. Col. phytosoc. 12: 249-267.

HOYOS, F. y A. BRAUN (s.f.) Palmas en Venezuela. Caracas.

HUBER, O. -1994- Recent advances in the phytogeography of the Guayana Region, South America. Mem. Soc. Biogeogr. 4: 5363.

HUBER, O., R. DUNO, R. RIINA, F. STAUFFER, L. PAPPATERRA, A. JIMÉNEZ, S. LLAMOZAS y G. ORSINI 1998- Estado actual del conocimiento de la flora en Venezuela. Caracas.

HUECK, K. -1966- Die Wälder Südamerikas. Ökologie, Zusammensetzung und wirtschafliche Bedeutung. Stuttgart.

KILLEEN, J., E. EMILIA GARCÍA \& S.G. BECK -1993- Guía de árboles de Bolivia. La 
Paz, St. Louis.

KNAPP, R. -1964- Höhere VegetationsEinheiten einiger Gebiete der holarktischen und neotropischen Floren-Reiche. Geobot. Mitteil. 28: 1-11.

KNAPP, R. -1980- Uber die Vegetation der Bermuda-Inseln II. Pflanzengesellschaften von Wäldern, Rasen und in HackfruchtBeständen mit einem Vergleich mit entsprechenden Assoziationen anderer Gebiete. Phytocoenologia 7: 475-491.

LANDOLT, E. -1999- Pleustonic communities with Lemnaceae in South America. Appl. Veg. Sci. $2:$ 7-16.

LASSER, T. -1969- Origen de las formaciones vegetales de nuestros llanos. Acta Bot. Ven. 4: 23-28.

LÓPEZ-PALACIOS, S. -1977-Flora de Venezuela. Verbenaceae. Mérida.

LOVELESS, A.R. -1960- The vegetation of Antigua. J. Ecol. 48: 495-527.

MORRONE, J.J. -2001-Biogeografía de América Latina y el Caribe.- M \& T.Manuales \& Tesis SEA, vol. 3. Zaragoza.

MÜLLER, G.K. \& P. GUTTE -1985- Beiträgue zur Kenntnis der Vegetation der Flussauen, Sümpfe und Gëwasser der zentralperuanischen Küstenregion. Wiss. $Z$. Karl-Marx-Univ. Leipzig, Math.-Naturwiss. R. 34: 410-429.

MÜLLER, M.J. -1982- Selected climatic data for a global set of standard stations for vegetation science. The Hague, Boston, London.

PEINADO, M., F. ALCARAZ \& J. DELGADILLO -1995- Syntaxonomy of some halophilous communities of North and Central America. Phytocoenologia 25: 2331.

PLONCZAK, M. -1989- Struktur und Entwicklungsdynamik eines Naturwaldes unter Konzessionsbewirtschaftung in den westlichen Llanos Venezuelas. Göttinger Beiträge zur Land- und Forstwirtschaft in den Tropen und Subtropen 43: 1-134.

PLONCZAK, M. -1998- Tipos de bosque y su presión de uso en Venezuela. Quebracho 6: 69-74.

PRANCE, G.T. -1973- Phytogeographic support for the theory of Pleistocene forest refuges in the Amazon Basin, based on evidence from distribution patterns in Caryocariaceae, Chrysobalanaceae, Dichapetalaceae and Lecythidaceae. Acta Amazonica 3: 5-28.

PRANCE, G.T. -1977- The phytogeographic subdivisions of Amazonia and their influence on the selection of biological reserves. In Prance, G.T. \& Elias, T.S.: Extinction is Forever. New York.

PRAT, H. -1935- Notes botaniques sur l'archipel des Bermudes. Bull. Soc. Bot. France 82: 162168.

RAMIA, M. -1959- Las Sabanas de Apure. Caracas.

RAMIA, M. -1967- Tipos de Sabanas en los Llanos de Venezuela. Bol. Soc. Ven. Ci. Nat. 27: 264288.

RAMIA, M. -1972- Cambios de vegetación de las sabanas del Hato El Frío (Alto Apure) causadas por diques. Bol. Soc. Ven. Ci. Nat. 30: 57-90.

RANGEL, O. -2004-El Chocó biogeográfico/ Costa Pacífica. Colombia Diversidad Biótica, $I V$ - Santafé de Bogotá.

RANGEL, O., P.D. LOWY y M. AGUILAR -1997Tipos de vegetación en Colombia. Colombia Diversidad Biótica, II-Santafé de Bogotá.

RIAL B., A. -2000- Aspectos cualitativos de la zonación y estratificación de comunidades de plantas acuáticas en un humedal de los Llanos de Venezuela. Mem. Fund. La Salle Ci. Nat. 60: 69-85.

RIVAS-MARTÍNEZ, S. -2005- Global Bioclimatics (www.globalbioclimatics.org).

RIVAS-MARTÍNEZ, S., D. SÁNCHEZ-MATA \& M. COSTA - 1999- North American Boreal and Western temperate Forest Vegetation (Syntaxonomical sinopsis of the potential natural plant communities of North America, II). Itinera Geobot. 12: 5-316.

SANTIAGO-VALENTÍN, E. \& R.G. OLMSTEAD, R.G. -2004- Historical biogeography of Caribbean plants: introduction to current knowledge and possibilities from a phylogenetic perspective. Taxon 53: 299-319.

SARMIENTO, G. \& M. MONASTERIO -1969Studies on the savanna vegetation of the Venezuelan Llanos. I. The use of associationanalysis. J. Ecol. 57: 169-598.

SEIBERT, P. -1996- Farbatlas Südamerika. Landschaften und Vegetation. Stuttgart. 
STEYERMARK, J.A. -1974- Rubiaceae. Flora de Venezuela. Caracas.

STEYERMARK, J.A., P. BERRY, H. BRUCE \& K. YATSKIEVYCH -1995-2005- Flora of the Venezuelan Guayana. St. Louis.

SUSACH CAMPALANS, F. -1989Caracterización y Clasificación Fitosociológica de la Vegetación de Sabanas del Sector Oriental de los Llanos Centrales Bajos Venezolanos. Acta Biol. Venez. 12: 154.

VAN DONSELAAR, J. -1965- The open-savanna vegetation. Class LeptocoryphioTrachypogonetea. Wentia 14: 84-131.

VÁSQUEZ MARTÍNEZ, R. -1997-Flórula de las Reservas Biológicas de Iquitos, Perú. St. Louis.

VELÁSQUEZ, J. -1994- Plantas acuáticas vasculares de Venezuela. Caracas.

VELASCO, A. y J. AYARZAGÜENA -1995Situación actual de las poblaciones de baba (Caiman crocodilus) sometidas al aprovechamiento comercial en los llanos venezolanos. Publ. As. Amigos Doñana 5: 571.

WALTER, H. -1997- Zonas de vegetación y clima. Barcelona.

WALTER, H. y E. MEDINA -1971Caracterización climática de Venezuela sobre la base de climadiagramas de estaciones particulares. Bol. Soc. Ven. Ci. Nat. 29: 211240.

WEBER, H.E., J. MORAVEC \& J.P. THEURILLAT -2000- International Code of Phytosociological Nomenclature. 3rd edition. J. Veg. Sci. 11: 739-768.
WIJNINGA, V.M., J. RANGEL \& A.M. CLEEF -1989- Botanical ecology and conservation of the Laguna de la Herrera (Sabana de Bogotá, Colombia). Caldasia 16(76): 23-40.

ZEILHOFER, P. \& M. SCHESSL -1999Relationship between vegetation and environmental conditions in the northern Pantanal of Mato Grosso, Brazil. J. Biogeogr. 27: 159-168.

Aceptado para su publicación en abril de 2006

Dirección de los autores. Departamento de Ciencias Ambientales y Recursos Naturales (Botánica), Facultad de Farmacia, Universidad San Pablo-CEU, Apartado 67, E- 28660 Boadilla del Monte, Madrid. 
